ESAIM: M2AN 55 (2021) 429-448

https://doi.org/10.1051/m2an/2020083
ESAIM: Mathematical Modelling and Numerical Analysis

www.esaim-m2an.org

\title{
NON-LOCAL VARIANT OF THE OPTIMISED SCHWARZ METHOD FOR ARBITRARY NON-OVERLAPPING SUBDOMAIN PARTITIONS
}

\author{
Xavier Claeys*
}

\begin{abstract}
We consider a scalar wave propagation in harmonic regime modelled by Helmholtz equation with heterogeneous coefficients. Using the Multi-Trace Formalism (MTF), we propose a new variant of the Optimized Schwarz Method (OSM) that remains valid in the presence of cross-points in the subdomain partition. This leads to the derivation of a strongly coercive formulation of our Helmholtz problem posed on the union of all interfaces. The corresponding operator takes the form "identity + non-expansive".
\end{abstract}

Mathematics Subject Classification. 65N38, 65N55, 31B10.

Received October 11, 2019. Accepted December 3, 2020.

\section{INTRODUCTION}

The effective solution to large scale wave propagation problems relates to a wide range of applications and yet remains a challenge, in particular when simulating highly oscillatory phenomena. With the growing importance of parallel computing, an intense research effort has been dedicated, in recent years, to the development of domain decomposition strategies that can be efficiently applied to wave propagation problems.

There is now a vast literature and a rich arsenal of well established domain decomposition techniques to deal with symmetric positive problems see e.g. $[31,48,54]$. By essence though, wave propagation does not fall into this symmetric positive framework and domain decomposition is much less developed for waves, from the point of view of both theory and effective numerical computation.

In the case of harmonic regime propagation, the Optimized Schwarz Method (OSM) appears to be one of the most effective available approaches for domain decomposition in a wave context. A general overview of this method and its numerous variants is given in [36]. In OSM, the coupling of subdomains is maintained through transmission conditions at interfaces, and these transmission conditions are formulated in terms of ingoing and outgoing trace operators involving impedance coefficients. The efficiency of OSM crucially depends on the choice of these impedances.

The Optimized Schwarz Method, originally proposed by Lions [44], was adapted by Després for wave propagation in [26-29] considering general non-overlapping partition of the computational domain and constant scalar impedance coefficients. Although, in such a general geometrical setting, OSM with scalar impedance was proved to converge, no assessment was provided as regards the rate of convergence. In practice, the convergence

Keywords and phrases. Domain decomposition, wave propagation, Helmholtz, integral operators, cross point.

Sorbonne Université, Université Paris-Diderot SPC, CNRS, Inria, Laboratoire Jacques-Louis Lions, équipe Alpines, Paris, France.

*Corresponding author: claeys@ann.jussieu.fr 


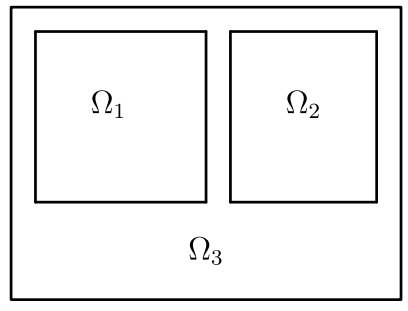

(a)

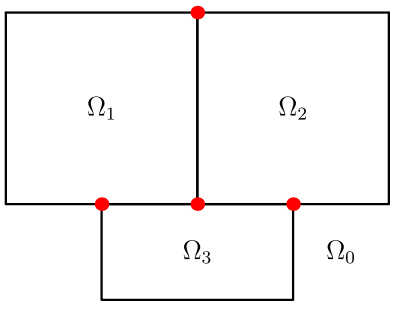

(b)

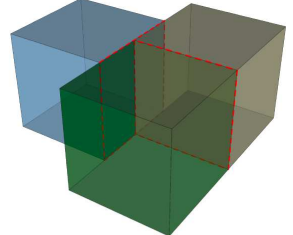

(c)

Figure 1. Examples of subdomain partitions in 2D (a \& b) and 3D (c) with 4 subdomains ( 3 bounded + exterior). There is no cross point in (a), and cross points are red dots in (b) and red dashed lines in (c).

could be slow. This was improved by Collino and Joly in $[20,21,42]$ where the authors proposed operator valued self-adjoint positive impedance coefficients and could establish geometric convergence of the method assuming that the subdomain partition does not involve any cross point i.e. point of adjacency of three interfaces (or one interface meeting the boundary of the compuational domain), see Figure 1 above. In another series of contributions Antoine, Geuzaine and their collaborators [2,3,32,33,55] considered the case of impedance coefficients approaching appropriate Dirichlet-to-Neumann maps and obtained fastly converging numerical methods. Here also, the numerical methods were observed to be of good quality only when the subdomain partition does not contain any cross point.

To deal with cross points, the literature dedicated to substructuring DDM already offers techniques such as dual-primal FETI (see e.g. [48], Chap. 5) which are purely discrete methods where so-called primal dofs located at cross points are kept unchanged, while the other dofs are teared apart in substructures. Each iteration of the DDM solver then requires inversion of the matrix coupling all primal dofs. Dual-primal FETI was adapted to wave propagation by Boubendir [7,9] by further augmenting local matrices to enforce the matching conditions in a way similar to the approach of Després. Energy estimates were established and led to a convergence result of DDM solvers although Section 4 in [7] pointed that the convergence rate could not be proved mesh-uniform.

Although Boubendir's work already provides an effective treatment, cross-points remained a thorny issue (see [34] for a thorough explanation) which recently attracted a renewed attention [4, 5, 30,35]. Very similar difficulties arise in a different context: the derivation of Boundary Integral Equations (BIE) adapted to multidomain scattering. The Multi-Trace Formalism (MTF) was introduced in $[11,12,14,16]$ as a complete framework for dealing with multi-domain BIE. From the perspective of functional analysis, MTF offers a clean treatment of cross-points. It would thus appear natural to try using the techniques developed in the Multi-Trace framework for dealing properly with cross points in Optimized Schwarz domain decomposition. This is precisely the aim of the present contribution.

In the present article, we rigorously establish equivalence bewteen a classical scattering problem in heterogeneous medium and a novel formulation (7.2) posed on the skeleton of a subdomain partition. This formulation is proved strongly coercive. We interpret Richardson's linear solver applied to this novel skeleton formulation as a new variant of the Optimized Schwarz Method. This new variant can be applied with any non-overlapping partition of the propagtion medium into Lipschitz subdomains, no matter the presence of cross-points. The key ingredient in this formulation is a non-local exchange operator used to enforce transmission conditions. Such exchange operator has always existed in previous versions of OSM, but it was so far systematically assumed to be a local operator consisting in swapping the traces from both sides of each interface of the subdomain partition. The exchange operator we consider here is more elaborate, which is the main novelty of our approach. The regularity assumptions that we formulate are rather loose regarding both material coefficients (piecewise 
Lipschitz) and geometry of subdomains (Lipschitz), which is another novelty made possible by our special exchange operator.

The formulation described in the present contribution cannot be considered as an extension of other preexisting OSM strategies including [7, 9, 20,21,42]. The difference lies in our new exchange operator that is non-local and does not coincide with the standard local operator that swaps traces from each side of each interface. Because of this non-locality, even subdomains that are not adjacent will be coupled, contrary to other OSM strategies.

It should be mentionned that the present contribution is purely analytical and that, in its present form, this new variant of OSM does not seem appropriate for actual numerical computations. This is why we do not report on numerical results. A discrete counterpart of the present strategy is presented in [15] where concrete numerical results are available. We still believe that the formulation we present here is an interesting theoretical object. In particular, it yields a strongly coercive formulation of Helmholtz problem which is not trivial: the derivation of coercive formulations for Helmoltz equation has been, in itself, the subject of recent attention [46].

In the case of piecewise constant material coefficients, formulation (7.2) can also be used as a multi-domain coupling scheme for the solution to scattering problems by means of boundary integral formulation. In the particular case of piecewise constant coefficients, the new formulation presented here can be considered as an alternative to other multi-domain BIE such as Multi-Trace [16], Boundary Element Tearing and Interconnecting [40], or Rumsey's reaction principle [56]. However we emphasize that the present contribution is not specifically oriented toward the derivation of a coupled boundary integral equation system, and a salient feature of the subsequent analysis lies in its ability to deal with heterogeneous propagation media.

\section{Geometry AND PROBlem Under STUdy}

In the present article, we are interested in a classical wave propagation problem in harmonic regime set in an heterogeneous medium in $\mathbb{R}^{d}$ for $d=1,2$ or 3 . We consider two essentially bounded measurable functions $\mu: \mathbb{R}^{d} \rightarrow \mathbb{R}_{+}$and $\kappa: \mathbb{R}^{d} \rightarrow \mathbb{C}_{+}$, and we assume that there exist constants $\kappa_{0}, \rho_{0}>0$ such that

$$
\begin{aligned}
& \text { (i) } \sup _{\boldsymbol{x} \in \mathbb{R}^{d}}\left(|\mu(\boldsymbol{x})|+\left|\mu^{-1}(\boldsymbol{x})\right|+|\kappa(\boldsymbol{x})|\right)<+\infty \text {. } \\
& \text { (ii) } \Re e\{\kappa(\boldsymbol{x})\} \geq 0, \Im m\{\kappa(\boldsymbol{x})\} \geq 0, \kappa(\boldsymbol{x}) \neq 0 \quad \forall \boldsymbol{x} \in \mathbb{R}^{d} . \\
& \text { (iii) } \kappa(\boldsymbol{x})=\kappa_{0} \text { and } \mu(\boldsymbol{x})=1 \text { for }|\boldsymbol{x}|>\rho_{0} .
\end{aligned}
$$

These assumptions are rather general yet reasonable enough to make the scattering problem we wish to examine properly well posed. We insist that we do not assume $\kappa, \mu$ to be piecewise constant. For some $f \in \mathrm{L}^{2}\left(\mathbb{R}^{d}\right)$ with bounded support, we wish to solve the following wave propagation problem in heterogeneous medium

$$
\left\{\begin{array}{l}
u \in \mathrm{H}_{\text {loc }}^{1}\left(\mathbb{R}^{d}\right) \text { such that } \\
-\operatorname{div}(\mu \nabla u)-\kappa^{2} u=f \quad \text { in } \mathbb{R}^{d}, \\
\lim _{\rho \rightarrow \infty} \int_{\partial \mathrm{B}_{\rho}}\left|\partial_{\rho} u-\imath \kappa_{0} u\right|^{2} \mathrm{~d} \sigma_{\rho}=0
\end{array}\right.
$$

where $\mathrm{B}_{\rho}$ refers to the ball of radius $\rho$ centered at $0, \sigma_{\rho}$ is the associated surface measure, and $\partial_{\rho}$ is the partial derivative with respect to $|\boldsymbol{x}|$. The third condition in (2.2) is usually referred to as Sommerfeld radiation condition. Well-posedness of the problem above is a classical result of scattering theory, see e.g. [43], Chapter 3 or [22], Chapter 7.

We wish to solve this problem by means of non-overlapping Domain Decomposition (DDM), which leads us to introduce a subdomain partitionning $\mathbb{R}^{d}=\cup_{j=0}^{\mathrm{J}} \bar{\Omega}_{j}$ with $\Omega_{j} \cap \Omega_{k}=\emptyset$ if $j \neq k$, each $\Omega_{j}$ is a Lipschitz domain, and $\Omega_{j}$ is bounded for $j \neq 0$. The "skeleton" will refer to the union of all interfaces between subdomains

$$
\Gamma=\partial \Omega_{0} \cup \cdots \cup \partial \Omega_{\mathrm{J}}
$$


We emphasize that such geometrical configuration allows the presence of junction points i.e. points where three subdomains or more abut. Examples of such non-overlapping multi-domain configurations are given in Figure 1.

For the sake of simplicity, we make further regularity assumptions on material coefficients in each subdomain, assuming that $\mu$ is Lipschitz regular in each subdomain,

$$
\begin{aligned}
& \nabla \mu_{j} \in \mathrm{L}^{\infty}\left(\Omega_{j}\right) \forall j=0 \ldots \mathrm{J}, \\
& \text { where } \mu_{j}:=\left.\mu\right|_{\Omega_{j}} .
\end{aligned}
$$

Assumptions (2.1)-(2.3) allow the coefficients $\mu, \kappa$ to jump across the interfaces $\partial \Omega_{j} \cap \partial \Omega_{k}$, but discards jumps of $\mu$ inside each subdomain. In particular, this setting includes the case where $\mu, \kappa$ are piecewise constant with respect to the subdomain partition.

Problem (2.2) can be decomposed according to the subdomain partition introduced above, leading to wave equations in each subdomain coupled by transmission conditions imposed through each interface

$$
\begin{aligned}
& \left\{\begin{array}{l}
u \in \mathrm{H}_{\mathrm{loc}}^{1}\left(\bar{\Omega}_{j}\right) \text { such that } \\
-\operatorname{div}(\mu \nabla u)-\kappa^{2} u=f \quad \text { in } \Omega_{j}, \\
\lim _{\rho \rightarrow \infty} \int_{\partial \mathrm{B}_{\rho}}\left|\partial_{\rho} u-\imath \kappa_{0} u\right|^{2} \mathrm{~d} \sigma_{\rho}=0,
\end{array}\right. \\
& \begin{cases}\left.u\right|_{\partial \Omega_{j}} ^{\mathrm{int}}-\left.u\right|_{\partial \Omega_{k}} ^{\mathrm{int}}=0 & \forall j, k=0 \ldots n \\
\left.\mu_{j} \partial_{n_{j}} u\right|_{\partial \Omega_{j}} ^{\mathrm{int}}+\left.\mu_{k} \partial_{n_{k}} u\right|_{\partial \Omega_{k}} ^{\mathrm{int}}=0 & \text { on } \partial \Omega_{j} \cap \partial \Omega_{k}\end{cases}
\end{aligned}
$$

where $\boldsymbol{n}_{j}$ refers to the normal vector field on $\partial \Omega_{j}$ directed toward the exterior of $\Omega_{j}$, and $\partial_{n_{j}} v:=\boldsymbol{n}_{j} \cdot \nabla v$. The boundary traces coming into play in the transmission conditions above are taken from the interior of the subdomains, which is the meaning of the "int" superscript.

The present contribution will consist in deriving a strongly coercive reformulation of problem (2.4) and (2.5) of the form "identity+contraction". This reformulation will be posed in a space of trace on the skeleton $\Gamma$.

From here until the end of Section 5, we are going to develop a functional toolkit for traces on the skeleton of our subdomain partition. Besides topological considerations (spaces, norms, ...), this framework involves two important ingredients: a global DtN map T (see Sect. 3.4) and a non-local exchange operator П (see Cor. 5.1). This framework is based on the Yukawa equation i.e. a PDE associated to the operator $-\Delta+\gamma^{-2}$ for some parameter $\gamma>0$. We emphasize that the analysis presented in Sections 3-5 is independent of the scattering problem (2.2) and that the parameter $\gamma$ is not a priori connected to material coefficients $\mu, \kappa$.

We will come back to our wave propagation problem in Sections 6 and 7 where, relying on this functional toolkit, we shall derive a reformulation of (2.2) and establish its well-posedness and strong coercivity.

\section{TRACE SPACES AND OPERATORS}

The treatment of interfaces between subdomains is a crucial aspect of any domain decomposition strategy, both for constructing or analysing it. As a consequence we pay a special attention to trace spaces.

\subsection{Volume based spaces}

First of all we need to fix a few notations related to classical volume based function spaces. For any Lipschitz domain $\Omega \subset \mathbb{R}^{d}$, the space $\mathrm{L}^{2}(\Omega)$ will refer to square integrable functions equipped with the norm $\|\varphi\|_{\mathrm{L}^{2}(\Omega)}^{2}:=$ $\int_{\Omega}|\varphi|^{2} \mathrm{~d} \boldsymbol{x}$. The Sobolev space $\mathrm{H}^{1}(\Omega):=\left\{\varphi \in \mathrm{L}^{2}(\Omega), \nabla \varphi \in \mathrm{L}^{2}(\Omega)^{d}\right\}$ will be equipped with the norm

$$
\|v\|_{\mathrm{H}^{1}(\Omega)}^{2}:=\|\nabla v\|_{\mathrm{L}^{2}(\Omega)}^{2}+\gamma^{-2}\|v\|_{\mathrm{L}^{2}(\Omega)}^{2} .
$$


In this definition $\gamma>0$ refers to a parameter that will be fixed all through this article. Occasionally we shall consider $\mathrm{H}(\operatorname{div}, \Omega):=\left\{\boldsymbol{\psi} \in \mathrm{L}^{2}(\Omega)^{d}, \operatorname{div}(\psi) \in \mathrm{L}^{2}(\Omega)\right\}$ and $\mathrm{H}^{1}(\Delta, \Omega):=\left\{\varphi \in \mathrm{H}^{1}(\Omega), \Delta \varphi \in \mathrm{L}^{2}(\Omega)\right\}$ equipped with the norm given by $\|\varphi\|_{\mathrm{H}^{1}(\Delta, \Omega)}^{2}:=\|\varphi\|_{\mathrm{H}^{1}(\Omega)}^{2}+\|\Delta \varphi\|_{\mathrm{L}^{2}(\Omega)}^{2}$. Finally if $\mathrm{H}(\Omega)$ refers to any of the spaces introduced above, then $\mathrm{H}_{\mathrm{loc}}(\bar{\Omega})$ shall refer to all functions $v: \Omega \rightarrow \mathbb{C}$ such that $v \varphi \in \mathrm{H}(\Omega)$ for all $\varphi \in \mathscr{C}_{\mathrm{comp}}^{\infty}\left(\mathbb{R}^{d}\right):=\left\{\psi \in \mathscr{C}^{\infty}\left(\mathbb{R}^{d}\right), \operatorname{supp}(\psi)\right.$ bounded $\}$.

\subsection{Traces on the boundary of a single subdomain}

Let us consider a Lipschitz open set $\Omega \subset \mathbb{R}^{d}$ such that either $\Omega$ or $\mathbb{R}^{d} \backslash \Omega$ is bounded. We shall refer to the space of Dirichlet traces $\mathrm{H}^{1 / 2}(\partial \Omega):=\left\{\left.v\right|_{\partial \Omega}, v \in \mathrm{H}^{1}(\Omega)\right\}$ equipped with the norm, see Theorem 3.40 of [45],

$$
\|v\|_{\mathrm{H}^{1 / 2}(\partial \Omega)}:=\min \left\{\|\varphi\|_{\mathrm{H}^{1}(\Omega)},\left.\varphi\right|_{\partial \Omega}=v\right\} .
$$

The space of Neumann traces $\mathrm{H}^{-1 / 2}(\partial \Omega)$ will be defined as the dual to $\mathrm{H}^{1 / 2}(\partial \Omega)$ equipped with the corresponding canonical dual norm $\|p\|_{\mathrm{H}^{-1 / 2}(\partial \Omega)}:=\sup _{v \in \mathrm{H}^{\lambda / e}(\partial \Omega) \backslash\{0\}}\left|\langle p, v\rangle_{\partial \Omega}\right| /\|v\|_{\mathrm{H}^{1 / 2}(\partial \Omega)}$. Here $v \mapsto\langle p, v\rangle_{\partial \Omega}:=p(v)$ simply refers to the action of $p$ on $v$, so that $(p, v) \mapsto\langle p, v\rangle_{\partial \Omega}$ is a bilinear (not sesquilinear) form. As regards duality pairing, we shall also equivalently write $\langle v, p\rangle_{\partial \Omega}:=\langle p, v\rangle_{\partial \Omega}$ and

$$
\int_{\partial \Omega} p v \mathrm{~d} \sigma=\langle p, v\rangle_{\partial \Omega}
$$

We will also equip the space of pairs of Dirichlet/Neumann traces with its own duality pairing. Although many choices are possible, we use a skew-symmetric pairing that appears naturally in energy conservation calculus, defined by

$$
\begin{aligned}
& {[(u, p),(v, q)]_{\partial \Omega}:=\langle u, q\rangle_{\partial \Omega}-\langle v, p\rangle_{\partial \Omega}} \\
& \text { for }(u, p) \text { and }(v, q) \text { in } \mathrm{H}^{+\frac{1}{2}}(\partial \Omega) \times \mathrm{H}^{-\frac{1}{2}}(\partial \Omega) .
\end{aligned}
$$

Note that this pairing does not involve any complex conjugation. Let $\boldsymbol{n}_{\Omega}$ refer to the normal vector field on $\partial \Omega$ directed toward the exterior of $\Omega$. As detailed in e.g. Theorem 2.21 and Lemma 4.4 of [53] or Theorem 3.38 and Lemma 4.3 of [45], each Lipschitz open set $\Omega \subset \mathbb{R}^{d}$ with bounded boundary gives rise to continuous operators $\tau_{\mathrm{D}}^{\Omega}: \mathrm{H}_{\mathrm{loc}}^{1}(\bar{\Omega}) \rightarrow \mathrm{H}^{1 / 2}(\partial \Omega), \tau_{\mathrm{N}}^{\Omega}: \mathrm{H}_{\mathrm{loc}}^{1}(\Delta, \bar{\Omega}) \rightarrow \mathrm{H}^{-1 / 2}(\partial \Omega)$ and $\tau^{\Omega}: \mathrm{H}_{\mathrm{loc}}^{1}(\Delta, \bar{\Omega}) \rightarrow \mathrm{H}^{1 / 2}(\partial \Omega) \times \mathrm{H}^{-1 / 2}(\partial \Omega)$ uniquely defined by

$$
\begin{array}{ll}
\tau_{\mathrm{D}}^{\Omega}(\varphi):=\left.\varphi\right|_{\partial \Omega} \quad \text { and } \quad \tau_{\mathrm{N}}^{\Omega}(\varphi):=\left.\boldsymbol{n}_{\Omega} \cdot \nabla \varphi\right|_{\partial \Omega}, \\
\tau^{\Omega}(\varphi):=\left(\tau_{\mathrm{D}}^{\Omega}(\varphi), \tau_{\mathrm{N}}^{\Omega}(\varphi)\right) \quad \forall \varphi \in \mathscr{C}^{\infty}(\bar{\Omega}) .
\end{array}
$$

Remark 3.1. In the case where $\Omega$ is one of the subdomains $\Omega_{j}, j=0 \ldots \mathrm{J}$, assume that some function $v \in$ $\mathrm{H}_{\text {loc }}^{1}\left(\bar{\Omega}_{j}\right)$ satisfies $\operatorname{div}(\mu \nabla v) \in \mathrm{L}_{\text {loc }}^{2}\left(\bar{\Omega}_{j}\right)$. Then according to (2.1) and (2.3), we have $\Delta v+\mu_{j}^{-1} \nabla \mu_{j} \cdot \nabla v \in \mathrm{L}_{\text {loc }}^{2}\left(\bar{\Omega}_{j}\right)$ and thus $\Delta v \in \mathrm{L}_{\text {loc }}^{2}\left(\bar{\Omega}_{j}\right)$. In particular if $u \in \mathrm{H}_{\text {loc }}^{1}\left(\bar{\Omega}_{j}\right)$ satisfies $\operatorname{div}(\mu \nabla u)+\kappa^{2} u=-f$ in $\Omega_{j}$ with $f$ as above, then $u \in \mathrm{H}_{\mathrm{loc}}^{1}\left(\Delta, \Omega_{j}\right)$ and its Dirichlet and Neumann traces in (3.4) are properly defined.

Remark 3.2. In the case where $\Omega$ is the exterior subdomain $\Omega_{0}$, according to previous paragraphs, the normal vector $\boldsymbol{n}_{0}$ is directed toward the exterior of $\Omega_{0}$ and the traces in (3.4) are taken from the interior of $\Omega_{0}$.

\subsection{Scalar products and Dirichlet-to-Neumann maps}

For any $v \in \mathrm{H}^{1 / 2}(\partial \Omega)$ let $\phi_{\mathrm{D}}(v) \in \mathrm{H}^{1}(\Omega)$ refer to the unique element that achieves the minimum in (3.2) i.e. such that $\|v\|_{\mathrm{H}^{1 / 2}(\partial \Omega)}=\left\|\phi_{\mathrm{D}}(v)\right\|_{\mathrm{H}^{1}(\Omega)}$. Writing Euler's identity for this minimisation problem, we see that $\int_{\Omega} \nabla \phi_{\mathrm{D}}(v) \cdot \nabla \varphi+\gamma^{-2} \phi_{\mathrm{D}}(v) \varphi \mathrm{d} \boldsymbol{x}=0 \forall \varphi \in \mathrm{H}_{0}^{1}(\Omega)$, which re-writes $-\Delta \phi_{\mathrm{D}}(v)+\gamma^{-2} \phi_{\mathrm{D}}(v)=0$ in $\Omega$. Then we introduce a so-called Dirichlet-to-Neumann $(\mathrm{DtN}) \operatorname{map} \mathrm{T}_{\Omega}:=\tau_{\mathrm{N}}^{\Omega} \cdot \phi_{\mathrm{D}}: \mathrm{H}^{1 / 2}(\partial \Omega) \rightarrow \mathrm{H}^{-1 / 2}(\partial \Omega)$, see e.g. [53], 
Section 6.6.3, [45], Chapter 4 or [48], Section 1.2.2.4. To be more explicit $\mathrm{T}_{\Omega}$ is defined by

$$
\begin{aligned}
& \mathrm{T}_{\Omega}(v):=\left.\boldsymbol{n}_{\Omega} \cdot \nabla \phi_{\mathrm{D}}(v)\right|_{\partial \Omega} \\
& \text { where } \phi_{\mathrm{D}}(v) \in \mathrm{H}^{1}(\Omega) \text { satisfies } \\
& \Delta \phi_{\mathrm{D}}(v)-\gamma^{-2} \phi_{\mathrm{D}}(v)=0 \quad \text { in } \Omega \\
& \left.\phi_{\mathrm{D}}(v)\right|_{\partial \Omega}=v \quad \text { on } \partial \Omega .
\end{aligned}
$$

This DtN map actually induces the scalar product associated to the norm (3.2). First of all observe that $\overline{\phi_{\mathrm{D}}(u)}=$ $\phi_{\mathrm{D}}(\bar{u})$ obviously, which implies $\overline{\mathrm{T}_{\Omega}(v)}=\mathrm{T}_{\Omega}(\bar{v})$. Next, according to the PDE satisfied by $\phi_{\mathrm{D}}$ in (3.5), applying Green's formula we obtain $\int_{\Omega} \nabla \phi_{\mathrm{D}}(u) \cdot \nabla \phi_{\mathrm{D}}(\bar{v})+\gamma^{-2} \phi_{\mathrm{D}}(u) \phi_{\mathrm{D}}(\bar{v}) \mathrm{d} \boldsymbol{x}=\int_{\partial \Omega} \phi_{\mathrm{D}}(\bar{v}) \boldsymbol{n}_{\Omega} \cdot \nabla \phi_{\mathrm{D}}(u) \mathrm{d} \sigma=\left\langle\mathrm{T}_{\Omega}(u), \bar{v}\right\rangle_{\partial \Omega}$. From this calculation it is clear that $\left\langle\mathrm{T}_{\Omega}(u), \bar{v}\right\rangle_{\partial \Omega}=\left\langle\mathrm{T}_{\Omega}(\bar{v}), u\right\rangle_{\partial \Omega}$ in other words $\mathrm{T}_{\Omega}$ is a self-adjoint operator. This property plays an important role in the definition of the scalar product we wish to adopt for the trace space $\mathrm{H}^{1 / 2}(\partial \Omega)$. Since by the defintion of $\phi_{\mathrm{D}}$ we have $\|u\|_{\mathrm{H}^{1 / 2}(\partial \Omega)}=\left\|\phi_{\mathrm{D}}(u)\right\|_{\mathrm{H}^{1}(\Omega)}$, we can take the following as scalar product on the Dirichlet trace spaces

$$
(u, v)_{\mathrm{H}^{1 / 2}(\partial \Omega)}:=\left\langle\mathrm{T}_{\Omega}(u), \bar{v}\right\rangle_{\partial \Omega} \quad \text { for } u, v \in \mathrm{H}^{1 / 2}(\partial \Omega) .
$$

According to Riesz representation theorem, for any $p \in \mathrm{H}^{-1 / 2}(\partial \Omega)$ there exists a unique $\varphi_{p} \in \mathrm{H}^{1 / 2}(\partial \Omega)$ such that $\langle p, v\rangle_{\partial \Omega}=\left(\varphi_{p}, v\right)_{\mathrm{H}^{1 / 2}(\partial \Omega)}=\left\langle\mathrm{T}_{\Omega}\left(\varphi_{p}\right), v\right\rangle$ for all $v \in \mathrm{H}^{1 / 2}(\partial \Omega)$. Hence $\varphi_{p}=\left(\mathrm{T}_{\Omega}\right)^{-1}(p)$ and $\|p\|_{\mathrm{H}^{-1 / 2}(\partial \Omega)}^{2}=$ $\left\|\varphi_{p}\right\|_{\mathrm{H}^{1 / 2}(\partial \Omega)}^{2}=\left\langle\mathrm{T}_{\Omega}\left(\varphi_{p}\right), \bar{\varphi}_{p}\right\rangle_{\partial \Omega}=\left\langle p, \mathrm{~T}_{\Omega}^{-1}(\bar{p})\right\rangle_{\partial \Omega}$. As a consequence the norm on Neumann data is induced by the following scalar product

$$
(p, q)_{\mathrm{H}^{-1 / 2}(\partial \Omega)}:=\left\langle p, \mathrm{~T}_{\Omega}^{-1}(\bar{q})\right\rangle_{\partial \Omega} \quad \text { for } p, q \in \mathrm{H}^{-1 / 2}(\partial \Omega) .
$$

\subsection{Traces in a multi-domain setting}

We will also need to consider cartesian products of Dirichlet or Neumann trace spaces based on the boundary of each subdomain of the partition, which we call multi-trace spaces defined as follows

$$
\begin{aligned}
& \mathbb{H}_{\mathrm{D}}(\Gamma):=\mathrm{H}^{+\frac{1}{2}}\left(\partial \Omega_{0}\right) \times \cdots \times \mathrm{H}^{+\frac{1}{2}}\left(\partial \Omega_{\mathrm{J}}\right) \\
& \mathbb{H}_{\mathrm{N}}(\Gamma):=\mathrm{H}^{-\frac{1}{2}}\left(\partial \Omega_{0}\right) \times \cdots \times \mathrm{H}^{-\frac{1}{2}}\left(\partial \Omega_{\mathrm{J}}\right) \\
& \mathbb{H}(\Gamma):=\Pi_{j=0 \ldots \mathrm{J}} \mathrm{H}^{+\frac{1}{2}}\left(\partial \Omega_{j}\right) \times \mathrm{H}^{-\frac{1}{2}}\left(\partial \Omega_{j}\right)
\end{aligned}
$$

equipped with $\|\mathfrak{p}\|_{\mathbb{H}_{N}(\Gamma)}^{2}:=\left\|p^{0}\right\|_{\mathrm{H}^{-1 / 2}\left(\partial \Omega_{0}\right)}^{2}+\cdots+\left\|p^{\mathrm{J}}\right\|_{\mathrm{H}^{-1 / 2}\left(\partial \Omega_{\mathrm{J}}\right)}^{2}$ for $\mathfrak{p}=\left(p^{j}\right)_{j=0}^{\mathrm{J}} \in \mathbb{H}_{\mathrm{N}}(\Gamma)$, and analogous definitions for \|\|$_{\mathbb{H}_{\mathbb{D}}(\Gamma)}$ and \|\|$_{\mathbb{H}(\Gamma)}$. The multi-trace space $\mathbb{H}(\Gamma)$ coincides with $\mathbb{H}_{D}(\Gamma) \times \mathbb{H}_{N}(\Gamma)$ through a reordering of traces which is why, when considering an element $\mathfrak{u}=\left(u_{\mathrm{D}}^{j}, u_{\mathrm{N}}^{j}\right)_{j=0}^{J} \in \mathbb{H}(\Gamma)$, we will sometimes commit a slight abuse of notation writing " $\mathfrak{u}=\left(\mathfrak{u}_{\mathrm{D}}, \mathfrak{u}_{\mathrm{N}}\right)$ " to refer to the Dirichlet components $\mathfrak{u}_{\mathrm{D}}=\left(u_{\mathrm{D}}^{j}\right)_{j=0}^{\mathrm{J}} \in \mathbb{H}_{\mathrm{D}}(\Gamma)$ on the one hand, and the Neumann components $\mathfrak{u}_{\mathrm{N}}=\left(u_{\mathrm{N}}^{j}\right)_{j=0}^{\mathrm{J}} \in \mathbb{H}_{\mathrm{N}}(\Gamma)$ on the other hand. There is a natural duality between Dirichlet and Neumann multi-trace spaces through the bilinear pairing

$$
\langle\langle\mathfrak{u}, \mathfrak{p}\rangle\rangle:=\sum_{j=0}^{\mathrm{J}}\left\langle u^{j}, p^{j}\right\rangle_{\partial \Omega_{j}} \quad \forall \mathfrak{u}=\left(u^{0}, \ldots, u^{\mathrm{J}}\right) \in \mathbb{H}_{\mathrm{D}}(\Gamma), \quad \forall \mathfrak{p}=\left(p^{0}, \ldots, p^{\mathrm{J}}\right) \in \mathbb{H}_{\mathrm{N}}(\Gamma) .
$$

The bilinear pairing defined above does not involve any complex conjugation operation. We shall indifferently write $\langle\langle\mathfrak{p}, \mathfrak{u}\rangle\rangle:=\langle\langle\mathfrak{u}, \mathfrak{p}\rangle\rangle$ for $\mathfrak{u} \in \mathbb{H}_{\mathrm{D}}(\Gamma), \mathfrak{p} \in \mathbb{H}_{\mathrm{N}}(\Gamma)$. 
For the sake of conciseness, we shall denote $\mathrm{T}_{j}$ instead of $\mathrm{T}_{\Omega_{j}}$. The operator $\mathrm{T}:=\operatorname{diag}_{j=0 \ldots \mathrm{J}}\left(\mathrm{T}_{j}\right): \mathbb{H}_{\mathrm{D}}(\Gamma) \rightarrow$ $\mathbb{H}_{\mathrm{N}}(\Gamma)$ induces a scalar product underlying the norm of $\mathbb{H}_{\mathrm{N}}(\Gamma)$ through

$$
\begin{aligned}
(\mathfrak{p}, \mathfrak{q})_{\mathbb{H}_{N}(\Gamma)} & =\left\langle\left\langle\mathrm{T}^{-1}(\mathfrak{p}), \overline{\mathfrak{q}}\right\rangle\right\rangle=\sum_{j=0}^{\mathrm{J}}\left\langle\mathrm{T}_{j}^{-1}\left(p^{j}\right), \bar{q}^{j}\right\rangle_{\partial \Omega_{j}} \\
& =\sum_{j=0}^{\mathrm{J}}\left(p^{j}, q^{j}\right)_{\mathrm{H}^{-1 / 2}\left(\partial \Omega_{j}\right)}
\end{aligned}
$$

for any $\mathfrak{p}=\left(p^{j}\right)_{j=0 \ldots \mathrm{J}}$ and any $\mathfrak{q}=\left(q^{j}\right)_{j=0 \ldots \mathrm{J}}$ in $\mathbb{H}_{\mathrm{N}}(\Gamma)$. As regards $\mathbb{H}(\Gamma)$, we shall consider a duality pairing given by the following skew symetric bilinear form

$$
\begin{aligned}
& \llbracket \mathfrak{u}, \mathfrak{v} \rrbracket:=\left[\mathfrak{u}_{0}, \mathfrak{v}_{0}\right]_{\partial \Omega_{0}}+\cdots+\left[\mathfrak{u}_{\mathrm{J}}, \mathfrak{v}_{\mathrm{J}}\right]_{\partial \Omega_{\mathrm{J}}} \\
& \text { for } \mathfrak{u}=\left(\mathfrak{u}_{j}\right)_{j=0}^{\mathrm{J}} \text { and } \mathfrak{v}=\left(\mathfrak{v}_{j}\right)_{j=0}^{\mathrm{J}} \text { in } \mathbb{H}(\Gamma) .
\end{aligned}
$$

As regards trace operators, for the sake of conciseness, we shall denote $\tau^{j}:=\tau^{\Omega_{j}}$ and adopt similar conventions for $\tau_{\mathrm{D}}^{j}$ and $\tau_{\mathrm{N}}^{j}$. We also introduce global trace operators that map into multi-trace spaces

$$
\begin{aligned}
& \tau_{\alpha}(u):=\left(\tau_{\alpha}^{0}(u), \ldots, \tau_{\alpha}^{\mathrm{J}}(u)\right) \quad \text { for } \alpha=\mathrm{D}, \mathrm{N} \\
& \tau(u):=\left(\tau^{0}(u), \ldots, \tau^{\mathrm{J}}(u)\right) .
\end{aligned}
$$

\section{Transmission COnditions}

Since we are considering a problem involving transmission conditions (2.5), it is natural to introduce the subspace of $\mathbb{H}(\Gamma)$ consisting in all tuples of traces agreeing with these conditions: this is what shall be called single-trace spaces defined by

$$
\begin{aligned}
& \mathbb{X}_{\mathrm{D}}(\Gamma):=\left\{\left(v_{j}\right)_{j=0}^{\mathrm{J}} \in \mathbb{H}_{\mathrm{D}}(\Gamma)\left|\exists \varphi \in \mathrm{H}^{1}\left(\mathbb{R}^{d}\right), v_{j}=\varphi\right|_{\partial \Omega_{j}} \forall j\right\} \\
& \mathbb{X}_{\mathrm{N}}(\Gamma):=\left\{\left(q_{j}\right)_{j=0}^{\mathrm{J}} \in \mathbb{H}_{\mathrm{N}}(\Gamma)\left|\exists \boldsymbol{\psi} \in \mathrm{H}\left(\operatorname{div}, \mathbb{R}^{d}\right), q_{j}=\boldsymbol{n}_{j} \cdot \boldsymbol{\psi}\right|_{\partial \Omega_{j}} \forall j\right\} \\
& \mathbb{X}(\Gamma):=\left\{\mathfrak{u}=\left(\mathfrak{u}_{\mathrm{D}}, \mathfrak{u}_{\mathrm{N}}\right) \in \mathbb{H}(\Gamma) \mid \mathfrak{u}_{\mathrm{D}} \in \mathbb{X}_{\mathrm{D}}(\Gamma), \mathfrak{u}_{\mathrm{N}} \in \mathbb{X}_{\mathrm{N}}(\Gamma)\right\} .
\end{aligned}
$$

By construction, for a function $u \in \mathrm{L}_{\text {loc }}^{2}\left(\mathbb{R}^{d}\right)$ such that $\left.u\right|_{\Omega_{j}} \in \mathrm{H}_{\text {loc }}^{1}\left(\Delta, \bar{\Omega}_{j}\right)$ for all $j=0 \ldots \mathrm{J}$, the transmission conditions $(2.5)$ are equivalent to the statement " $\tau(u) \in \mathbb{X}(\Gamma)$ ", in the case where $\mu_{0}=\ldots=\mu_{\mathrm{J}}$. The single-trace space has been extensively studied in the context of multi-trace formulations [14]. The following caracterisation of this space was proved in Proposition 6.3 of [13].

Proposition 4.1. For any $\mathfrak{u} \in \mathbb{H}(\Gamma)$ we have $\mathfrak{u} \in \mathbb{X}(\Gamma) \Longleftrightarrow \llbracket \mathfrak{u}, \mathfrak{v} \rrbracket=0 \forall \mathfrak{v} \in \mathbb{X}(\Gamma)$.

Proof. From (4.1), it is clear that any $\mathfrak{u}=\left(\mathfrak{u}_{\mathrm{D}}, \mathfrak{u}_{\mathrm{N}}\right) \in \mathbb{H}(\Gamma)$ actually belongs to $\mathbb{X}(\Gamma)$ if and only if $\mathfrak{u}_{\mathrm{D}} \in \mathbb{X}_{\mathrm{D}}(\Gamma)$ and $\mathfrak{u}_{\mathrm{N}} \in \mathbb{X}_{\mathrm{N}}(\Gamma)$. As a consequence, to prove the lemma, it suffices to show that for any $\mathfrak{u}_{\mathrm{D}} \in \mathbb{H}_{\mathrm{D}}(\Gamma)$ and any $\mathfrak{u}_{\mathrm{N}} \in \mathbb{H}_{\mathrm{N}}(\Gamma)$ we have

(i) $\mathfrak{u}_{\mathrm{D}} \in \mathbb{X}_{\mathrm{D}}(\Gamma) \Longleftrightarrow\left\langle\left\langle\mathfrak{u}_{\mathrm{D}}, \mathfrak{q}\right\rangle\right\rangle=0 \forall \mathfrak{q} \in \mathbb{X}_{\mathrm{N}}(\Gamma)$.

(ii) $\mathfrak{u}_{\mathrm{N}} \in \mathbb{X}_{\mathrm{N}}(\Gamma) \Longleftrightarrow\left\langle\left\langle\mathfrak{u}_{\mathrm{N}}, \mathfrak{v}\right\rangle\right\rangle=0 \forall \mathfrak{v} \in \mathbb{X}_{\mathrm{D}}(\Gamma)$.

We will only present the proof of (i) since the proof for (ii) is very similar. Take an arbitrary $\mathfrak{u}_{\mathrm{D}}=\left(u_{\mathrm{D}}^{j}\right)_{j=0}^{\mathrm{J}} \in$ $\mathbb{H}_{\mathrm{D}}(\Gamma)$. If $\mathfrak{u}_{\mathrm{D}} \in \mathbb{X}_{\mathrm{D}}(\Gamma)$, there exists $\varphi \in \mathrm{H}^{1}\left(\mathbb{R}^{d}\right)$ such that $\left.\varphi\right|_{\partial \Omega_{j}}=u_{\mathrm{D}}^{j} \forall j=0 \ldots \mathrm{J}$. Then for any $\mathfrak{q}=\left(q^{j}\right)_{j=0}^{\mathrm{J}} \in$ $\mathbb{X}_{\mathrm{N}}(\Gamma)$, there exists $\boldsymbol{\psi} \in \mathrm{H}\left(\operatorname{div}, \mathbb{R}^{d}\right)$ such that $\left.\boldsymbol{n}_{j} \cdot \boldsymbol{\psi}\right|_{\partial \Omega_{j}}=q^{j} \forall j=0 \ldots$ J. Applying a Green formula in each $\Omega_{j}$ 
on the one hand, and in $\mathbb{R}^{d}$ on the other hand, we obtain

$$
\begin{aligned}
\left\langle\left\langle\mathfrak{u}_{\mathrm{D}}, \mathfrak{q}\right\rangle\right\rangle & =\sum_{j=0}^{\mathrm{J}}\left\langle u_{\mathrm{D}}^{j}, q^{j}\right\rangle_{\partial \Omega_{j}}=\sum_{j=0}^{\mathrm{J}} \int_{\partial \Omega_{j}} \boldsymbol{n}_{j} \cdot \boldsymbol{\psi} \varphi \mathrm{d} \sigma \\
& =\sum_{j=0}^{\mathrm{J}} \int_{\Omega_{j}} \nabla \varphi \cdot \boldsymbol{\psi}+\varphi \operatorname{div} \boldsymbol{\psi} \mathrm{d} \boldsymbol{x}=\int_{\mathbb{R}^{d}} \nabla \boldsymbol{\varphi} \cdot \boldsymbol{\psi}+\varphi \operatorname{div} \boldsymbol{\psi} \mathrm{d} \boldsymbol{x}=0 .
\end{aligned}
$$

Now assume that $\mathfrak{u}_{\mathrm{D}}=\left(u_{\mathrm{D}}^{0}, \ldots, u_{\mathrm{D}}^{\mathrm{J}}\right) \in \mathbb{H}_{\mathrm{D}}(\Gamma)$ satisfies $\left\langle\left\langle\mathfrak{u}_{\mathrm{D}}, \mathfrak{q}\right\rangle\right\rangle=0 \forall \mathfrak{q} \in \mathbb{X}_{\mathrm{N}}(\Gamma)$. For each $j=0 \ldots \mathrm{J}$, introduce a lifting $v_{j} \in \mathrm{H}^{1}\left(\Omega_{j}\right)$ such that $\left.v_{j}\right|_{\partial \Omega_{j}}=u_{\mathrm{D}}^{j}$, and set $v(\boldsymbol{x})=1_{\Omega_{0}}(\boldsymbol{x}) v_{0}(\boldsymbol{x})+\cdots+1_{\Omega_{\mathrm{J}}}(\boldsymbol{x}) v_{\mathrm{J}}(\boldsymbol{x})$. We have clearly $v \in \mathrm{L}^{2}\left(\mathbb{R}^{d}\right)$ and, to prove that $\mathfrak{u}_{\mathrm{D}} \in \mathbb{X}_{\mathrm{D}}(\Gamma)$, it suffices to show that $v \in \mathrm{H}^{1}\left(\mathbb{R}^{d}\right)$. Define $\boldsymbol{p} \in \mathrm{L}^{2}\left(\mathbb{R}^{d}\right)$ by $\boldsymbol{p}(\boldsymbol{x})=1_{\Omega_{0}}(\boldsymbol{x}) \nabla v_{0}(\boldsymbol{x})+\cdots+1_{\Omega_{\mathrm{J}}}(\boldsymbol{x}) \nabla v_{\mathrm{J}}(\boldsymbol{x})$. Pick an arbitrary $\boldsymbol{\psi} \in \mathrm{H}\left(\operatorname{div}, \mathbb{R}^{d}\right)$, and set $\mathfrak{q}=\left(q^{j}\right)_{j=0}^{\mathrm{J}}$ where $q^{j}:=\left.\boldsymbol{n}_{j} \cdot \boldsymbol{\psi}\right|_{\partial \Omega_{j}}$. Since $\mathfrak{q} \in \mathbb{X}_{\mathrm{N}}(\Gamma)$, we have

$$
\begin{aligned}
\int_{\mathbb{R}^{d}} v \operatorname{div}(\boldsymbol{\psi}) \mathrm{d} \boldsymbol{x} & =\sum_{j=0}^{\mathrm{J}} \int_{\Omega_{j}} v \operatorname{div}(\boldsymbol{\psi}) \mathrm{d} \boldsymbol{x} \\
& =\left\langle\left\langle\mathfrak{u}_{\mathrm{D}}, \mathfrak{q}\right\rangle\right\rangle-\sum_{j=0}^{\mathrm{J}} \int_{\Omega_{j}} \boldsymbol{\psi} \cdot \nabla v_{j} \mathrm{~d} \boldsymbol{x} \\
& =-\int_{\mathbb{R}^{d}} \boldsymbol{\psi} \cdot \boldsymbol{p} \mathrm{d} \boldsymbol{x} .
\end{aligned}
$$

Since the above identity holds for any $\boldsymbol{\psi} \in \mathrm{H}\left(\operatorname{div}, \mathbb{R}^{d}\right)$, we conclude that $v$ admits a weak gradient over $\mathbb{R}^{d}$ as a whole with $\boldsymbol{p}=\nabla v$ in $\mathbb{R}^{d}$ and, as a consequence $v \in \mathrm{H}^{1}\left(\mathbb{R}^{d}\right)$ and $\mathfrak{u}_{\mathrm{D}} \in \mathbb{X}_{\mathrm{D}}(\Gamma)$.

As underlined during its proof, the above caracterisation implies that $\mathfrak{u} \in \mathbb{H}_{D}(\Gamma)$ belongs to $\mathbb{X}_{D}(\Gamma)$ if and only if $\langle\langle\mathfrak{u}, \mathfrak{p}\rangle\rangle=0 \forall \mathfrak{p} \in \mathbb{X}_{\mathrm{N}}(\Gamma)$ and that, similarly, $\mathfrak{p} \in \mathbb{H}_{\mathrm{N}}(\Gamma)$ belongs to $\mathbb{X}_{\mathrm{N}}(\Gamma)$ if and only if $\langle\langle\mathfrak{u}, \mathfrak{p}\rangle\rangle=0 \forall \mathfrak{u} \in \mathbb{X}_{\mathrm{D}}(\Gamma)$. The next result provides a new variant of the decomposition established in Proposition 6.1 of [14].

Proposition 4.2. We have the direct sum $\mathbb{H}_{\mathrm{N}}(\Gamma)=\mathbb{X}_{\mathrm{N}}(\Gamma) \oplus \mathrm{T}\left(\mathbb{X}_{\mathrm{D}}(\Gamma)\right)$ and it is orthogonal with respect to the scalar product induced by $\mathrm{T}^{-1}$.

Proof. First, according to Proposition 4.1, we have $(\mathfrak{p}, \mathrm{T}(\mathfrak{u}))_{\mathbb{H}_{\mathbb{N}}(\Gamma)}=\langle\langle\mathfrak{p}, \mathfrak{u}\rangle\rangle=0$ whenever $\mathfrak{p} \in \mathbb{X}_{\mathrm{N}}(\Gamma)$ and $\mathfrak{u} \in \mathbb{X}_{\mathrm{D}}(\Gamma)$. This proves that $\mathbb{X}_{N}(\Gamma)$ is orthogonal to $\mathrm{T}\left(\mathbb{X}_{\mathrm{D}}(\Gamma)\right)$ hence $\mathbb{X}_{N}(\Gamma) \cap \mathrm{T}\left(\mathbb{X}_{\mathrm{D}}(\Gamma)\right)=\{0\}$.

Next pick an arbitrary $\mathfrak{p} \in \mathbb{H}_{\mathrm{N}}(\Gamma)$ and, by Riesz representation theorem, define $\mathfrak{u}$ as the unique element of $\mathbb{X}_{\mathrm{D}}(\Gamma)$ satisfying $\langle\langle\mathrm{T}(\mathfrak{u}), \overline{\mathfrak{v}}\rangle\rangle=\langle\langle\mathfrak{p}, \overline{\mathfrak{v}}\rangle\rangle$ for all $\mathfrak{v} \in \mathbb{X}_{\mathrm{D}}(\Gamma)$. As a consequence $\mathfrak{q}=\mathfrak{p}-\mathrm{T}(\mathfrak{u})$ satisfies $\langle\langle\mathfrak{q}, \mathfrak{v}\rangle\rangle=0 \forall \mathfrak{v} \in \mathbb{X}_{\mathrm{D}}(\Gamma)$ and thus belongs to $\mathbb{X}_{\mathrm{N}}(\Gamma)$ according to Proposition 4.1. This shows that $\mathbb{H}_{\mathrm{N}}(\Gamma)=\mathbb{X}_{\mathrm{N}}(\Gamma)$ $+\mathrm{T}\left(\mathbb{X}_{\mathrm{D}}(\Gamma)\right)$.

\section{Potential theory}

The problem (2.2) primarily considered in the present manuscript does not a priori lend itself to boundary integral equation techniques simply because (2.2) is a problem of propagation in heterogeneous media i.e. the PDEs involve a priori varying coefficients. However several aspects of the solution strategy we wish to describe involve nonlocal operators. In particular, we shall need such theoretical tools for treatment of junctions. As a consequence, we dedicate the present section to recalling a few facts about boundary integral operators. 


\subsection{Layer potentials in a single subdomain}

We first introduce the Green kernel $\mathscr{G}(\boldsymbol{x})$ of the Yukawa's equation i.e. we define $\mathscr{G}$ as the unique function solving $-\Delta \mathscr{G}+\gamma^{-2} \mathscr{G}=\delta_{0}$ in $\mathbb{R}^{d}$ and $\lim _{|\boldsymbol{x}| \rightarrow \infty} \mathscr{G}(\boldsymbol{x})=0$, where $\delta_{0}$ is the Dirac measure centered at $\boldsymbol{x}=0$, and $\gamma>0$ is a parameter that we have fixed once and for all in Section 3.1. This kernel admits an explicit expression in terms of special functions namely

$$
\begin{array}{lll}
\mathscr{G}(\boldsymbol{x}):=\mathrm{K}_{0}(|\boldsymbol{x}| / \gamma), & \boldsymbol{x} \in \mathbb{R}^{2} \backslash\{0\} & \text { for } d=2, \\
\mathscr{G}(\boldsymbol{x}):=\frac{\exp (-|\boldsymbol{x}| / \gamma)}{4 \pi|\boldsymbol{x}|}, & \boldsymbol{x} \in \mathbb{R}^{3} \backslash\{0\} & \text { for } d=3
\end{array}
$$

where $\mathrm{K}_{0}$ refers to the modified Bessel function of the second kind of order 0 also known as MacDonald function, see Section 10.25 of [47]. With this kernel, and for any Lipschitz domain $\Omega \subset \mathbb{R}^{d}$ with bounded boundary, we can define single and double layer potentials as follows: for any $(v, q) \in \mathrm{H}^{1 / 2}(\partial \Omega) \times \mathrm{H}^{-1 / 2}(\partial \Omega)$ we set

$$
\begin{aligned}
& \Psi^{\Omega}(v, q)(\boldsymbol{x}):=\Psi_{\mathrm{D}}^{\Omega}(v)(\boldsymbol{x})+\Psi_{\mathrm{N}}^{\Omega}(q)(\boldsymbol{x}), \\
& \text { where } \quad \Psi_{\mathrm{D}}^{\Omega}(v)(\boldsymbol{x}):=\int_{\partial \Omega} \boldsymbol{n}_{\Omega}(\boldsymbol{y}) \cdot(\nabla \mathscr{G})(\boldsymbol{x}-\boldsymbol{y}) v(\boldsymbol{y}) \mathrm{d} \sigma(\boldsymbol{y}), \\
& \Psi_{\mathrm{N}}^{\Omega}(q)(\boldsymbol{x}):=\int_{\partial \Omega} \mathscr{G}(\boldsymbol{x}-\boldsymbol{y}) q(\boldsymbol{y}) \mathrm{d} \sigma(\boldsymbol{y}),
\end{aligned}
$$

for all $\boldsymbol{x} \in \mathbb{R}^{d} \backslash \partial \Omega$. For any $\mathfrak{v} \in \mathrm{H}^{1 / 2}(\partial \Omega) \times \mathrm{H}^{-1 / 2}(\partial \Omega)$, we have $\left(\gamma^{-2}-\Delta\right) \Psi^{\Omega}(\mathfrak{v})=0$ both in $\Omega$ and $\mathbb{R}^{d} \backslash \bar{\Omega}$. Besides $\left.\Psi^{\Omega}(\mathfrak{v})\right|_{\Omega} \in \mathrm{H}^{1}(\Delta, \overline{\mathcal{O}})$ for $\mathcal{O}=\Omega$ or $\mathcal{O}=\mathbb{R}^{d} \backslash \bar{\Omega}$. For any $\boldsymbol{x}, \boldsymbol{y} \in \mathbb{R}^{d}, \boldsymbol{x} \neq \boldsymbol{y}$, define $\mathscr{G}_{\boldsymbol{x}}: \mathbb{R}^{d} \backslash\{\boldsymbol{x}\} \rightarrow \mathbb{R}_{+}$by $\mathscr{G}_{\boldsymbol{x}}(\boldsymbol{y}):=\mathscr{G}(\boldsymbol{x}-\boldsymbol{y})$. Elementary calculus shows that $\Psi^{\Omega}(\mathfrak{u})(\boldsymbol{x})=\left[\tau^{\Omega}\left(\mathscr{G}_{\boldsymbol{x}}\right), \mathfrak{u}\right]_{\partial \Omega}$ for all $\mathfrak{u} \in \mathrm{H}^{1 / 2}(\partial \Omega) \times \mathrm{H}^{-1 / 2}(\partial \Omega)$ and all $\boldsymbol{x} \in \mathbb{R}^{d} \backslash \partial \Omega$. The next result, known as representation theorem, shows that layer potential can be used to reconstruct any solution to the homogeneous Yukawa equation. A proof can be found in Lemma 3.4 of [24], Theorem 6.10 of [45], Theorem 3.1.8 of [52] or formula (5.2) of [53].

Proposition 5.1. For any Lipschitz domain $\Omega \subset \mathbb{R}^{d}$ with bounded boundary, and any function $u \in \mathrm{H}^{1}(\Omega)$ satisfying $\left(\gamma^{-2}-\Delta\right) u=0$ in $\Omega$, we have $\Psi^{\Omega}\left(\tau^{\Omega}(u)\right)=1_{\Omega}(\boldsymbol{x}) u(\boldsymbol{x}) \forall \boldsymbol{x} \in \mathbb{R}^{d}$.

Here $1_{\Omega}(\boldsymbol{x})=1$ if $\boldsymbol{x} \in \Omega$ and $1_{\Omega}(\boldsymbol{x})=0$ otherwise. In the representation formula above, the traces of solutions to the homogeneous PDE play a pivotal role. The potential operators actually provide a Calderón projector that maps onto such a space and can thus be used to caracterise them. A proof of the next result can be found in Proposition 3.6.2 of [52] or Lemma 6.18 of [53].

Proposition 5.2. The operator $\tau^{\Omega} \cdot \Psi^{\Omega}: \mathrm{H}^{1 / 2}(\partial \Omega) \times \mathrm{H}^{-1 / 2}(\partial \Omega) \rightarrow \mathrm{H}^{1 / 2}(\partial \Omega) \times \mathrm{H}^{-1 / 2}(\partial \Omega)$ is a continuous projector whose range is the space $\mathcal{C}_{\mathrm{in}}(\Omega):=\left\{\tau^{\Omega}(u) \mid u \in \mathrm{H}^{1}(\Omega),\left(\gamma^{-2}-\Delta\right) u=0\right.$ in $\left.\Omega\right\}$.

\subsection{Layer potentials in a multi-domain setting}

We now establish a few results about potential theory that are specific to the multi-domain context. Part of the present section is a variant of results already available in $[10,11]$. However there are novelties. In particular we consider here a strongly coercive equation as opposed to the heterogeneous indefinite problem of [10], which leads to stronger results and a completely new formulation of transmission conditions, see Proposition 5.4.

Considering $\Omega=\Omega_{j}$ for $j=0 \ldots \mathrm{J}$, the result of the previous paragraph can be used directly in the multidomain context. For the sake of conciseness, in the following, we shall write $\Psi_{\mathrm{D}}^{j}, \Psi_{\mathrm{N}}^{j}, \Psi^{j}$ instead of $\Psi_{\mathrm{D}}^{\Omega_{j}}, \Psi_{\mathrm{N}}^{\Omega_{j}}, \Psi^{\Omega_{j}}$.

We first show that an explicit formula for the orthogonal projector onto $\mathbb{X}_{\mathrm{N}}(\Gamma)$, can be obtained. We rely on so-called multi-potential operators $\Psi_{\mathrm{D}}: \mathbb{H}_{\mathrm{D}}(\Gamma) \rightarrow \Pi_{j=0}^{\mathrm{J}} \mathrm{H}_{\mathrm{loc}}^{1}\left(\Delta, \bar{\Omega}_{j}\right)$ and $\Psi_{\mathrm{N}}: \mathbb{H}_{\mathrm{N}}(\Gamma) \rightarrow \Pi_{j=0}^{\mathrm{J}} \mathrm{H}_{\mathrm{loc}}^{1}\left(\Delta, \bar{\Omega}_{j}\right)$ defined 
as follows: for any $\mathfrak{u}=\left(\mathfrak{u}_{\mathrm{D}}, \mathfrak{u}_{\mathrm{N}}\right) \in \mathbb{H}(\Gamma)$ we set

$$
\begin{aligned}
& \Psi(\mathfrak{u})(\boldsymbol{x})=\Psi_{\mathrm{D}}\left(\mathfrak{u}_{\mathrm{D}}\right)(\boldsymbol{x})+\Psi_{\mathrm{N}}\left(\mathfrak{u}_{\mathrm{N}}\right)(\boldsymbol{x}) \\
& \text { where } \quad \Psi_{\mathrm{D}}\left(\mathfrak{u}_{\mathrm{D}}\right)(\boldsymbol{x}):=\sum_{j=0}^{\mathrm{J}} \Psi_{\mathrm{D}}^{j}\left(u_{\mathrm{D}}^{j}\right)(\boldsymbol{x}), \\
& \Psi_{\mathrm{N}}\left(\mathfrak{u}_{\mathrm{N}}\right)(\boldsymbol{x}):=\sum_{j=0}^{\mathrm{J}} \Psi_{\mathrm{N}}^{j}\left(u_{\mathrm{N}}^{j}\right)(\boldsymbol{x})
\end{aligned}
$$

for any $\boldsymbol{x} \in \mathbb{R}^{d} \backslash \Gamma$. Such operators have been first considered in the context of the integral formulation of the second kind introduced in [10], see also [11,17-19,37,49,50]. The multi-potential operators satisfy many non-trivial properties. To begin with, the next proposition shows that they are closely related to global Dirichletto-Neumann maps.

Lemma 5.1. For any $\mathfrak{u}=\left(\mathfrak{u}_{\mathrm{D}}, \mathfrak{u}_{\mathrm{N}}\right) \in \mathbb{H}(\Gamma)$ we have $\tau \cdot \Psi(\mathfrak{u})=\mathfrak{u} \Longleftrightarrow \mathfrak{u}_{\mathrm{N}}=\mathrm{T}\left(\mathfrak{u}_{\mathrm{D}}\right)$.

Proof. Pick a $\mathfrak{u}=\left(\mathfrak{u}_{\mathrm{D}}, \mathfrak{u}_{\mathrm{N}}\right)=\left(\mathfrak{u}_{\mathrm{D}}^{j}, \mathfrak{u}_{\mathrm{N}}^{j}\right)_{j=0}^{J} \in \mathbb{H}(\Gamma)$ with $\mathfrak{u}_{\mathrm{N}}=\mathrm{T}\left(\mathfrak{u}_{\mathrm{D}}\right)$. We have $\mathfrak{u}^{j}:=\left(\mathfrak{u}_{\mathrm{D}}^{j}, \mathfrak{u}_{\mathrm{N}}^{j}\right)=\left(\mathfrak{u}_{\mathrm{D}}^{j}, \mathrm{~T}_{j}\left(\mathfrak{u}_{\mathrm{D}}^{j}\right)\right) \in$ $\mathcal{C}_{\text {in }}\left(\Omega_{j}\right)$ for each $j=0 \ldots$ J. As a consequence, applying Proposition 5.1, we obtain $\tau^{k} \Psi^{j}\left(\mathfrak{u}^{j}\right)=\delta_{j, k} \mathfrak{u}^{j}$ for any $j, k=0 \ldots \mathrm{J}$. Summing the latter identity over $j$ yields $\tau^{k} \Psi(\mathfrak{u})=\mathfrak{u}^{k}$ for all $k=0 \ldots \mathrm{J}$.

Let us now assume that $\mathfrak{u}=\left(\mathfrak{u}_{\mathrm{D}}, \mathfrak{u}_{\mathrm{N}}\right)=\left(\mathfrak{u}_{\mathrm{D}}^{j}, \mathfrak{u}_{\mathrm{N}}^{j}\right)_{j=0}^{\mathrm{J}} \in \mathbb{H}(\Gamma)$ satisfies $\tau \cdot \Psi(\mathfrak{u})=\mathfrak{u}$. By construction, the function $\psi:=\Psi(\mathfrak{u})$ is well defined in $\mathbb{R}^{d} \backslash \Gamma$, it satisfies $\left(\gamma^{-2}-\Delta\right) \psi=0$ in each $\Omega_{j}, j=0 \ldots \mathrm{J}$ by definition of the potential operators $\Psi^{j}$ 's. It also satisfies $\tau_{\mathrm{D}}^{j}(\psi)=\mathfrak{u}_{\mathrm{D}}^{j}$ and $\tau_{\mathrm{N}}^{j}(\psi)=\mathfrak{u}_{\mathrm{N}}^{j}$ for all $j=0 \ldots \mathrm{J}$ since $\tau \cdot \Psi(\mathfrak{u})=\mathfrak{u}$. By definition of the DtN maps given in Sections 3.3 and 3.4, this can be re-written $\mathfrak{u}_{\mathrm{N}}^{j}=\mathrm{T}_{j}\left(\mathfrak{u}_{\mathrm{D}}^{j}\right) \forall j=0 \ldots$ J. Put in vector form, this yields $\mathfrak{u}_{\mathrm{N}}=\mathrm{T}\left(\mathfrak{u}_{\mathrm{D}}\right)$.

The proof of the following result closely follows Lemma 5.1 of [10] and Lemma 6.3 of [11].

Lemma 5.2. We have $\Psi(\mathfrak{u})=0 \forall \mathfrak{u} \in \mathbb{X}(\Gamma)$.

Proof. Denoting as before $\mathscr{G}_{\boldsymbol{x}}(\boldsymbol{y}):=\mathscr{G}(\boldsymbol{x}-\boldsymbol{y})$, recall that we have $\Psi^{\Omega}(\mathfrak{u})(\boldsymbol{x})=\left[\tau^{\Omega}\left(\mathscr{G}_{\boldsymbol{x}}\right), \mathfrak{u}\right]_{\partial \Omega} \mathfrak{u} \in \mathrm{H}^{1 / 2}(\partial \Omega) \times$ $\mathrm{H}^{-1 / 2}(\partial \Omega)$ and all $\boldsymbol{x} \in \mathbb{R}^{d} \backslash \partial \Omega$. Plugging this expression into the definition of the multi-potential operator yields $\Psi(\mathfrak{u})(\boldsymbol{x})=\llbracket \tau\left(\mathscr{G}_{\boldsymbol{x}}\right), \mathfrak{u} \rrbracket \quad \forall \mathfrak{u} \in \mathbb{H}(\Gamma), \quad \forall \boldsymbol{x} \in \mathbb{R}^{d} \backslash \Gamma$. Now observe that for any $\boldsymbol{x} \in \mathbb{R}^{d} \backslash \Gamma$ we have $\tau\left(\mathscr{G}_{\boldsymbol{x}}\right) \in \mathbb{X}(\Gamma)$ hence applying Proposition 4.1 concludes the proof.

A direct consequence of the lemma above is that $\Psi_{\mathrm{D}}\left(\mathfrak{u}_{\mathrm{D}}\right)=0$ for all $\mathfrak{u}_{\mathrm{D}} \in \mathbb{X}_{\mathrm{D}}(\Gamma)$, and $\Psi_{\mathrm{N}}\left(\mathfrak{u}_{\mathrm{N}}\right)=0$ for all $\mathfrak{u}_{\mathrm{N}} \in \mathbb{X}_{\mathrm{N}}(\Gamma)$. We deduce in particular that $\mathbb{X}_{\mathrm{N}}(\Gamma) \subset \operatorname{Ker}\left(\tau_{\mathrm{N}} \cdot \Psi_{\mathrm{N}}\right)$. The proof of the next result is similar to that of Lemma 6.4 from [11].

Lemma 5.3. We have $\mathfrak{p}-\tau_{\mathrm{N}} \cdot \Psi_{\mathrm{N}}(\mathfrak{p}) \in \mathbb{X}_{\mathrm{N}}(\Gamma)$ for any $\mathfrak{p} \in \mathbb{H}_{\mathrm{N}}(\Gamma)$.

Proof. Pick an arbitrary $\mathfrak{p} \in \mathbb{H}_{N}(\Gamma)$ and, applying Proposition 4.2, decompose it as $\mathfrak{p}=\mathfrak{v}_{N}+\mathrm{T}\left(\mathfrak{u}_{\mathrm{D}}\right)$ where $\mathfrak{u}_{\mathrm{D}} \in \mathbb{X}_{\mathrm{D}}(\Gamma)$ and $\mathfrak{v}_{\mathrm{N}} \in \mathbb{X}_{\mathrm{N}}(\Gamma)$. According to Lemma 5.2 we have $\Psi_{\mathrm{D}}\left(\mathfrak{u}_{\mathrm{D}}\right)=0$ so that, setting $\mathfrak{u}:=\left(\mathfrak{u}_{\mathrm{D}}, \mathrm{T}\left(\mathfrak{u}_{\mathrm{D}}\right)\right)$, we have $\mathfrak{p}-\tau_{\mathrm{N}} \cdot \Psi_{\mathrm{N}}(\mathfrak{p})=\mathfrak{v}_{\mathrm{N}}-\tau_{\mathrm{N}} \cdot \Psi_{\mathrm{N}}\left(\mathfrak{v}_{\mathrm{N}}\right)+\mathrm{T}\left(\mathfrak{u}_{\mathrm{D}}\right)-\tau_{\mathrm{N}} \cdot \Psi(\mathfrak{u})$. Applying Lemma 5.1 yields $\mathrm{T}\left(\mathfrak{u}_{\mathrm{D}}\right)-\tau_{\mathrm{N}} \cdot \Psi(\mathfrak{u})=0$. Besides we have $\Psi_{\mathrm{N}}\left(\mathfrak{v}_{\mathrm{N}}\right)=0$ according to Lemma 5.2 since $\mathfrak{v}_{\mathrm{N}} \in \mathbb{X}_{\mathrm{N}}(\Gamma)$. To summarise, we have just established $\mathfrak{p}-\tau_{\mathrm{N}} \cdot \Psi_{\mathrm{N}}(\mathfrak{p})=\mathfrak{v}_{\mathrm{N}} \in \mathbb{X}_{\mathrm{N}}(\Gamma)$, which concludes the proof.

Combining the previous two lemmas, we see that $\left(\tau_{\mathrm{N}} \cdot \Psi_{\mathrm{N}}\right)\left(\mathrm{Id}-\tau_{\mathrm{N}} \cdot \Psi_{\mathrm{N}}\right)=0$. From this we deduce immediately the following proposition, which is a variant of Corollary 6.1 from [11].

Proposition 5.3. We have $\operatorname{Ker}\left(\tau_{\mathrm{N}} \cdot \Psi_{\mathrm{N}}\right)=\operatorname{Range}\left(\operatorname{Id}-\tau_{\mathrm{N}} \cdot \Psi_{\mathrm{N}}\right)=\mathbb{X}_{\mathrm{N}}(\Gamma)$, and $\tau_{\mathrm{N}} \cdot \Psi_{\mathrm{N}}: \mathbb{H}_{\mathrm{N}}(\Gamma) \rightarrow \mathbb{H}_{\mathrm{N}}(\Gamma)$ is a continuous projector. 
The next result is new and gives further details about the image of this projector.

Lemma 5.4. We have Range $\left(\tau_{\mathrm{N}} \cdot \Psi_{\mathrm{N}}\right)=\mathrm{T}\left(\mathbb{X}_{\mathrm{D}}(\Gamma)\right)$ so that $\tau_{\mathrm{N}} \cdot \Psi_{\mathrm{N}}$ is an orthogonal projector with respect to the scalar product induced by $\mathrm{T}^{-1}$ over $\mathbb{H}_{\mathrm{N}}(\Gamma)$.

Proof. Taking account of both Propositions 4.2 and 5.3, we see that it suffices to prove $\tau_{\mathrm{N}} \cdot \Psi_{\mathrm{N}}(\mathrm{T}(\mathfrak{u}))=\mathrm{T}(\mathfrak{u})$ for all $\mathfrak{u} \in \mathbb{X}_{\mathrm{D}}(\Gamma)$. Hence consider any $\mathfrak{u}=\left(u_{j}\right)_{j=0}^{\mathrm{J}} \in \mathbb{X}_{\mathrm{D}}(\Gamma)$. According to Lemma 5.2 we have $\tau_{\mathrm{N}} \cdot \Psi_{\mathrm{D}}(\mathfrak{u})=0$. As a consequence, applying Proposition 5.1, we obtain

$$
\begin{aligned}
\tau_{\mathrm{N}}^{k} \cdot \Psi_{\mathrm{N}}(\mathrm{T}(\mathfrak{u})) & =\tau_{\mathrm{N}}^{k} \cdot\left(\Psi_{\mathrm{D}}(\mathfrak{u})+\Psi_{\mathrm{N}}(\mathrm{T}(\mathfrak{u}))\right) \\
& =\tau_{\mathrm{N}}^{k} \cdot \sum_{j=0}^{\mathrm{J}} \Psi_{\mathrm{D}}^{j}\left(u_{j}\right)+\Psi_{\mathrm{N}}^{j}\left(\mathrm{~T}_{j}\left(u_{j}\right)\right) \\
& =\sum_{j=0}^{\mathrm{J}} \tau_{\mathrm{N}}^{k} \cdot \Psi_{\mathrm{D}}^{j}\left(u_{j}\right)+\tau_{\mathrm{N}}^{k} \cdot \Psi_{\mathrm{N}}^{j}\left(\mathrm{~T}_{j}\left(u_{j}\right)\right)=\mathrm{T}_{k}\left(u_{k}\right)
\end{aligned}
$$

for any $k=0 \ldots \mathrm{J}$. Since this holds for all $k$, we obtain that $\tau_{\mathrm{N}} \cdot \Psi_{\mathrm{N}}(\mathrm{T}(\mathfrak{u}))=\mathrm{T}(\mathfrak{u})$, which concludes the proof.

From the previous results, we immediately obtain an estimate on the norm of the projection, which will be key in the analysis of Section 7. Although it shares similarities with Proposition 6.1 of [11], the next result is new.

Corollary 5.1. Define $\Pi:=\operatorname{Id}-2 \tau_{\mathrm{N}} \cdot \Psi_{\mathrm{N}}$. Then we have $\Pi^{2}=\mathrm{Id}$ and the operators $(\mathrm{Id} \pm \Pi) / 2$ are continuous projectors with $\mathbb{X}_{\mathrm{N}}(\Gamma):=\operatorname{Ker}(\mathrm{Id}-\Pi)$ and $\mathrm{T}\left(\mathbb{X}_{\mathrm{D}}(\Gamma)\right):=\operatorname{Ker}(\mathrm{Id}+\Pi)$. Besides the following continuity estimate holds:

$$
\|\Pi(\mathfrak{p})\|_{\mathbb{H}_{N}(\Gamma)}=\|\mathfrak{p}\|_{\mathbb{H}_{N}(\Gamma)} \quad \forall \mathfrak{p} \in \mathbb{H}_{\mathrm{N}}(\Gamma) .
$$

Remark 5.1. The definition of $\Pi$ stems from an orthogonal projection with respect to the scalar product induced by the operator $\mathrm{T}$ defined in Section 3.4. So the operator $\Pi$ is tightly related to $\mathrm{T}$. This is an important fact and we will come back to this later.

In the subsequent analysis, this projector will be the key tool for caracterising elements of $\mathbb{X}(\Gamma)$ and thus enforcing transmission conditions across interfaces. The next result indeed provides a caracterisation of the single trace space that is a core novelty of the present contribution.

Proposition 5.4. Consider any $\omega>0$. With the notations of the previous corollary, for any $\mathfrak{u}=\left(\mathfrak{u}_{\mathrm{D}}, \mathfrak{u}_{\mathrm{N}}\right) \in$ $\mathbb{H}(\Gamma)$, we have $\mathfrak{u} \in \mathbb{X}(\Gamma)$ if and only if $\mathfrak{u}_{\mathrm{N}}-\imath \omega \mathrm{T}\left(\mathfrak{u}_{\mathrm{D}}\right)=\Pi\left(\mathfrak{u}_{\mathrm{N}}+\imath \omega \mathrm{T}\left(\mathfrak{u}_{\mathrm{D}}\right)\right)$.

Proof. According to Corollary 5.1, for $\mathfrak{u}=\left(\mathfrak{u}_{\mathrm{D}}, \mathfrak{u}_{\mathrm{N}}\right) \in \mathbb{H}(\Gamma)$, we have $\mathfrak{u}_{\mathrm{N}} \in \mathbb{X}_{\mathrm{N}}(\Gamma) \Longleftrightarrow(\mathrm{Id}-\Pi) \mathfrak{u}_{\mathrm{N}}=0$ and $\mathfrak{u}_{\mathrm{D}} \in \mathbb{X}_{\mathrm{D}}(\Gamma) \Longleftrightarrow(\mathrm{Id}+\Pi) \mathrm{T}\left(\mathfrak{u}_{\mathrm{D}}\right)=0$. On the other hand, Range $(\mathrm{Id}+\Pi) \cap$ Range $(\mathrm{Id}-\Pi)=\{0\}$ since $(\mathrm{Id}+\Pi) / 2$ is a projector, which leads to $\mathfrak{u} \in \mathbb{X}(\Gamma) \Longleftrightarrow(\mathrm{Id}-\Pi) \mathfrak{u}_{\mathrm{N}}=\imath \omega(\mathrm{Id}+\Pi) \mathrm{T}\left(\mathfrak{u}_{\mathrm{D}}\right)$. Rearranging this latter identity yields the conclusion of the proof.

\section{Reformulation of WAVE EQUATions}

In this section we focus on the wave equations (2.4) that we will reformulate in terms of traces only. We adopt the approach developed by Collino, Ghanemi and Joly in [21] and further studied and extended in [41,42]. This 
approach generalises the original work of Després [26-29] on Optimised Schwarz Method for Helmholtz equation. In the present section, we will derive a convenient caracterisation of

$$
\begin{array}{r}
\mathscr{C}^{+}(\Gamma):=\mathscr{C}^{+}\left(\Omega_{0}\right) \times \cdots \times \mathscr{C}^{+}\left(\Omega_{\mathrm{J}}\right) \text { where } \\
\mathscr{C}^{+}\left(\Omega_{j}\right):=\left\{\left(\tau_{\mathrm{D}}^{j}(\varphi), \mu_{j} \tau_{\mathrm{N}}^{j}(\varphi)\right) \in \mathrm{H}^{1 / 2}\left(\partial \Omega_{j}\right) \times \mathrm{H}^{-1 / 2}\left(\partial \Omega_{j}\right),\right. \\
\operatorname{div}(\mu \nabla \varphi)+\kappa^{2} \varphi=0 \text { in } \Omega_{j} \text { and } \\
\left.\varphi \kappa_{0}-\text { outgoing if } j=0\right\} .
\end{array}
$$

The space $\mathscr{C}^{+}\left(\Omega_{j}\right)$ is closed in $\mathrm{H}^{1 / 2}\left(\partial \Omega_{j}\right) \times \mathrm{H}^{-1 / 2}\left(\partial \Omega_{j}\right)$ and we will use these spaces to reformulate the wave equation in each subdomain. The following proposition provides an important decomposition of the multi-trace space. Although it was already established in Proposition 6.1 of [14] based on arguments of potential theory that assume piecewise homogeneity of the propagation medium, below is a new proof that also applies in the case of heterogeneous media.

Proposition 6.1. We have the direct sum $\mathbb{H}(\Gamma)=\mathbb{X}(\Gamma) \oplus \mathscr{C}^{+}(\Gamma)$.

Proof. Let us first show that $\mathbb{X}(\Gamma) \cap \mathscr{C}^{+}(\Gamma)=\{0\}$. Pick some $\mathfrak{u} \in \mathbb{X}(\Gamma) \cap \mathscr{C}^{+}(\Gamma)$ decomposed in Dirichlet/Neumann components $\mathfrak{u}=\left(\mathfrak{u}_{\mathrm{D}}, \mathfrak{u}_{\mathrm{N}}\right)$ with $\mathfrak{u}_{\mathrm{D}}=\left(u_{\mathrm{D}}^{j}\right)_{j=0}^{\mathrm{J}} \in \mathbb{H}_{\mathrm{D}}(\Gamma)$ and $\mathfrak{u}_{\mathrm{N}}=\left(u_{\mathrm{N}}^{j}\right)_{j=0}^{\mathrm{J}} \in \mathbb{H}_{\mathrm{N}}(\Gamma)$. For each $j=0 \ldots \mathrm{J}$, let $\phi_{j} \in \mathrm{H}_{\mathrm{loc}}^{1}\left(\Omega_{j}\right)$ refer to the unique functions satisfying

$$
\begin{aligned}
& \operatorname{div}\left(\mu \nabla \phi_{j}\right)+\kappa^{2} \phi_{j}=0 \quad \text { in } \Omega_{j}, \\
& \phi_{0} \text { is } \kappa_{0} \text {-outgoing, } \\
& \left(\tau_{\mathrm{D}}^{j}\left(\phi_{j}\right), \mu_{j} \tau_{\mathrm{N}}^{j}(\phi)\right)=\left(u_{\mathrm{D}}^{j}, u_{\mathrm{N}}^{j}\right) \text { on } \partial \Omega_{j} .
\end{aligned}
$$

Set $\phi:=1_{\Omega_{0}} \phi_{0}+\cdots+1_{\Omega_{\mathrm{J}}} \phi_{\mathrm{J}}$, so that $\operatorname{div}(\mu \nabla \phi)+\kappa^{2} \phi=0$ in each $\Omega_{j}$ and, since $\mathfrak{u}=\left(\tau_{\mathrm{D}}^{j}(\phi), \mu_{j} \tau_{\mathrm{N}}^{j}(\phi)\right)_{j=0 \ldots \mathrm{J}} \in \mathbb{X}(\Gamma)$ the function $\phi$ satisfies transmission conditions across $\Gamma$, so that $\operatorname{div}(\mu \nabla \phi)+\kappa^{2} \phi=0$ in $\mathbb{R}^{d}$ and $\phi$ is $\kappa_{0}$-outgoing. Well-posedness of the Helmholtz equation with outgoing radiation condition leads to $\phi=0$, hence $\mathfrak{u}=0$, which proves that

$$
\mathbb{X}(\Gamma) \cap \mathscr{C}^{+}(\Gamma)=\{0\}
$$

Now let us consider the general case of an arbitrary $\mathfrak{u} \in \mathbb{H}(\Gamma)$. Consider any lifting function $\psi^{\prime} \in \mathrm{L}^{2}\left(\mathbb{R}^{d}\right)$ with compact support such that $\left.\psi^{\prime}\right|_{\Omega_{j}} \in \mathrm{H}^{1}\left(\Omega_{j}\right)$ and $\tau_{\mathrm{D}}^{j}\left(\psi^{\prime}\right)=u_{\mathrm{D}}^{j}$ for all $j=0 \ldots \mathrm{J}$. Next define $\psi \in \mathrm{H}_{\mathrm{loc}}^{1}\left(\mathbb{R}^{d}\right)$ as the unique element of $\mathrm{H}_{\text {loc }}^{1}\left(\mathbb{R}^{d}\right)$ satisfying

$$
\begin{array}{r}
\sum_{j=0}^{\mathrm{J}} \int_{\Omega_{j}} \mu \nabla\left(\psi+\psi^{\prime}\right) \cdot \nabla \varphi-\kappa^{2}\left(\psi+\psi^{\prime}\right) \varphi \mathrm{d} \boldsymbol{x}=\left\langle\left\langle\mathfrak{u}_{\mathrm{N}}, \tau_{\mathrm{D}}(\varphi)\right\rangle\right\rangle \quad \forall \varphi \in \mathrm{H}_{\text {comp }}^{1}\left(\mathbb{R}^{d}\right) \\
\text { and } \lim _{\rho \rightarrow \infty} \int_{\partial \mathrm{B}_{\rho}}\left|\partial_{\rho} \psi-\imath \kappa_{0} \psi\right|^{2} \mathrm{~d} \sigma_{\rho}=0
\end{array}
$$

where $\mathrm{H}_{\text {comp }}^{1}\left(\mathbb{R}^{d}\right)$ refers to the elements of $\mathrm{H}^{1}\left(\mathbb{R}^{d}\right)$ that are boundedly supported. Existence and uniqueness of such a $\psi$ stems from well posedness of Helmholtz problems in unbounded heterogeneous media, see e.g. [22], Chapter 3. Applying a Green formula in each $\Omega_{j}$, we obtain

$$
\begin{aligned}
& \operatorname{div}\left(\mu \nabla\left(\psi+\psi^{\prime}\right)\right)+\kappa^{2}\left(\psi+\psi^{\prime}\right)=0 \quad \text { in each } \Omega_{j}, j=0 \ldots \mathrm{J} \\
& \lim _{\rho \rightarrow \infty} \int_{\partial \mathrm{B}_{\rho}}\left|\partial_{\rho} \psi-\imath \kappa_{0} \psi\right|^{2} \mathrm{~d} \sigma_{\rho}=0 .
\end{aligned}
$$

Setting $\mathfrak{v}=\left(\tau_{\mathrm{D}}^{j}\left(\psi+\psi^{\prime}\right), \mu_{j} \tau_{\mathrm{N}}^{j}\left(\psi+\psi^{\prime}\right)\right)_{j=0, \ldots, \mathrm{J}}$, the equations above imply that $\mathfrak{v} \in \mathscr{C}^{+}(\Gamma)$. Decomposing in Dirichlet/Neumann contributions $\mathfrak{v}=\left(\mathfrak{v}_{\mathrm{D}}, \mathfrak{v}_{\mathrm{N}}\right)$, we have $\mathfrak{v}_{\mathrm{D}}-\mathfrak{u}_{\mathrm{D}}=\left(\tau_{\mathrm{D}}^{j}(\psi)\right)_{j=0}^{\mathrm{J}} \in \mathbb{X}_{\mathrm{D}}(\Gamma)$ since $\psi \in \mathrm{H}_{\mathrm{loc}}^{1}\left(\mathbb{R}^{d}\right)$. 
Moreover, applying Green formulas once more in (6.4), we see $\left\langle\left\langle\mathfrak{v}_{\mathrm{N}}, \tau_{\mathrm{D}}(\varphi)\right\rangle\right\rangle=\left\langle\left\langle\mathfrak{u}_{\mathrm{N}}, \tau_{\mathrm{D}}(\varphi)\right\rangle\right\rangle$ for all $\varphi \in \mathrm{H}^{1}\left(\mathbb{R}^{d}\right)$. Using the weak caracterisation of single trace spaces given by Proposition 4.1 , we conclude that $\mathfrak{u}_{\mathrm{D}}-\mathfrak{v}_{\mathrm{D}} \in \mathbb{X}_{\mathrm{D}}(\Gamma)$ and $\mathfrak{u}_{\mathrm{N}}-\mathfrak{v}_{\mathrm{N}} \in \mathbb{X}_{\mathrm{N}}(\Gamma)$ hence, setting $\mathfrak{w}:=\mathfrak{u}-\mathfrak{v} \in \mathbb{X}(\Gamma)$, so that, with the decomposition $\mathfrak{u}=\mathfrak{v}+\mathfrak{w}$, we have established $\mathbb{H}(\Gamma)=\mathbb{X}(\Gamma)+\mathscr{C}^{+}(\Gamma)$ which, together with (6.3), concludes the proof.

Let us underline that the previous proposition does not require unique solvability of local sub-problems. It only assumes existence and uniqueness of the global scattering problem. This decomposition result can be regarded as analogous to Proposition 4.2 although, in the result above, the direct sum is a priori not orthogonal.

The next property relates to energy conservation considerations and will thus play a key role in the forthcoming convergence analysis. Although such a result is classical in scattering theory (see e.g. [23], Thm. 3.12), the next lemma is rephrased so as to fit our framework. We also provide a proof for the sake of completeness.

Lemma 6.1. We have $\imath[\mathfrak{u}, \overline{\mathfrak{u}}]_{\partial \Omega_{j}} \leq 0, \forall \mathfrak{u} \in \mathscr{C}^{+}\left(\Omega_{j}\right) \forall j=0$. .J. and thus $\imath \llbracket \mathfrak{u}, \overline{\mathfrak{u}} \rrbracket \leq 0 \forall \mathfrak{u} \in \mathscr{C}^{+}(\Gamma)$.

Proof. For any $\mathfrak{u} \in \mathrm{H}^{1 / 2}\left(\partial \Omega_{j}\right) \times \mathrm{H}^{-1 / 2}\left(\partial \Omega_{j}\right)$, let $\varphi \in \mathrm{H}_{\mathrm{loc}}^{1}\left(\bar{\Omega}_{j}\right)$ satisfy $\operatorname{div}(\mu \nabla \varphi)+\kappa^{2} \varphi=0$ in $\Omega_{j}$ and $\left(\tau_{\mathrm{D}}^{j}(\varphi), \mu_{j} \tau_{\mathrm{N}}^{j}(\varphi)\right)=\mathfrak{u}$ on $\partial \Omega_{j}$. For all $j=0 \ldots \mathrm{J}$, we have $\imath[\mathfrak{u}, \overline{\mathfrak{u}}]_{\partial \Omega_{j}}=2 \Im m\left\{\int_{\partial \Omega_{j}} \mu_{j} \tau_{\mathrm{N}}^{j}(\varphi) \tau_{\mathrm{D}}^{j}(\bar{\varphi}) \mathrm{d} \sigma\right\}$. In the case where $j \neq 0$, the domain $\Omega_{j}$ is bounded so that we can apply a simple Green formula on the later identity,

$$
\begin{aligned}
\imath[\mathfrak{u}, \overline{\mathfrak{u}}]_{\partial \Omega_{j}} & =2 \Im m\left\{\int_{\partial \Omega_{j}} \mu_{j} \tau_{\mathrm{N}}^{j}(\varphi) \tau_{\mathrm{D}}^{j}(\bar{\varphi}) \mathrm{d} \sigma\right\}=2 \Im m\left\{\int_{\Omega_{j}} \bar{\varphi} \operatorname{div}(\mu \nabla \varphi)+\mu|\nabla \varphi|^{2} \mathrm{~d} \boldsymbol{x}\right\} \\
& =2 \Im m\left\{\int_{\Omega_{j}} \mu|\nabla \varphi|^{2}-\kappa^{2}|\varphi|^{2} \mathrm{~d} \boldsymbol{x}\right\}=-2 \int_{\Omega_{j}} \Im m\left\{\kappa^{2}\right\}|\varphi|^{2} \mathrm{~d} \boldsymbol{x} \leq 0 .
\end{aligned}
$$

Note that, in the above inequality, we used that assumptions (2.1) imply $\Im m\left\{\kappa^{2}\right\} \geq 0$. In the case of $\Omega_{0}$ take any radius $\rho_{0}>0$ large enough to guarantee $\mathbb{R}^{d} \backslash \Omega_{0} \subset \mathrm{B}_{\rho_{0}}$. We can apply the same calculus as above, considering $\mathrm{B}_{\rho} \cap \Omega_{0}$ instead of $\Omega_{0}$. Taking account of the radiation condition satisfied by $\varphi(\boldsymbol{x})$ for $|\boldsymbol{x}| \rightarrow \infty$, and the fact that $\Im m\left\{\kappa^{2}\right\}$ is boundedly supported (since $\kappa(\boldsymbol{x})=\kappa_{0}$ for $|\boldsymbol{x}|>\rho_{0}$ ), we obtain

$$
\begin{aligned}
\imath[\mathfrak{u}, \overline{\mathfrak{u}}]_{\partial \Omega_{j}} & =-2 \int_{\Omega_{0}} \Im m\left\{\kappa^{2}\right\}|\varphi|^{2} \mathrm{~d} \boldsymbol{x}+2 \Im m\left\{\int_{\partial \mathrm{B}_{\rho}} \varphi \partial_{\rho} \bar{\varphi} \mathrm{d} \sigma\right\} \\
& \leq 2 \Im m\left\{\int_{\partial \mathrm{B}_{\rho}} \varphi \partial_{\rho} \bar{\varphi} \mathrm{d} \sigma\right\}=-\frac{1}{\kappa_{0}} \int_{\partial \mathrm{B}_{\rho}} 2 \Re e\left\{\imath \kappa_{0} \varphi \partial_{\rho} \bar{\varphi}\right\} \mathrm{d} \sigma \\
& =\frac{1}{\kappa_{0}} \int_{\partial \mathrm{B}_{\rho}}\left|\partial_{\rho} \varphi-\imath \kappa_{0} \varphi\right|^{2} \mathrm{~d} \sigma-\frac{1}{\kappa_{0}} \int_{\partial \mathrm{B}_{\rho}}\left|\partial_{\rho} \varphi\right|^{2} \mathrm{~d} \sigma-\kappa_{0} \int_{\partial \mathrm{B}_{\rho}}|\varphi|^{2} \mathrm{~d} \sigma \\
& \leq \frac{1}{\kappa_{0}} \int_{\partial \mathrm{B}_{\rho}}\left|\partial_{\rho} \varphi-\imath \kappa_{0} \varphi\right|^{2} \mathrm{~d} \sigma \quad \forall \rho>\rho_{0} \\
& \leq \liminf _{\rho \rightarrow \infty} \frac{1}{\kappa_{0}} \int_{\partial \mathrm{B}_{\rho}}\left|\partial_{\rho} \varphi-\imath \kappa_{0} \varphi\right|^{2} \mathrm{~d} \sigma=0 .
\end{aligned}
$$

\subsection{Robin trace operators}

The caracterisation of $\mathbb{X}(\Gamma)$ provided by Proposition 5.4 involved specific combinations of Neumann and Dirichlet trace operators. Let us bring the attention of the reader to the following elementary identity: for any $\mathfrak{v}=\left(\mathfrak{v}_{\mathrm{D}}, \mathfrak{v}_{\mathrm{N}}\right) \in \mathbb{H}(\Gamma)$, and any $\omega>0$ we have

$$
\begin{aligned}
\left\|\mathfrak{v}_{\mathrm{N}}+\imath \alpha \mathrm{T}\left(\mathfrak{v}_{\mathrm{D}}\right)\right\|_{\mathbb{H}_{\mathrm{N}}(\Gamma)}^{2} & =\left\|\mathfrak{v}_{\mathrm{N}}\right\|_{\mathbb{H}_{\mathrm{N}}(\Gamma)}^{2}+\omega^{2}\left\|\mathfrak{v}_{\mathrm{D}}\right\|_{\mathbb{H}_{\mathrm{D}}(\Gamma)}^{2}+2 \alpha \Re e\left\{\imath\left\langle\left\langle\mathfrak{v}_{\mathrm{D}}, \overline{\mathfrak{v}}_{\mathrm{N}}\right\rangle\right\rangle\right\} \\
& =\left\|\mathfrak{v}_{\mathrm{N}}\right\|_{\mathbb{H}_{\mathrm{N}}(\Gamma)}^{2}+\omega^{2}\left\|\mathfrak{v}_{\mathrm{D}}\right\|_{\mathbb{H}_{\mathrm{D}}(\Gamma)}^{2}-2 \alpha \Im m\left\{\left\langle\left\langle\mathfrak{v}_{\mathrm{D}}, \overline{\mathfrak{v}}_{\mathrm{N}}\right\rangle\right\rangle\right\} \\
& =\left\|\mathfrak{v}_{\mathrm{N}}\right\|_{\mathbb{H}_{\mathrm{N}}(\Gamma)}^{2}+\omega^{2}\left\|\mathfrak{v}_{\mathrm{D}}\right\|_{\mathbb{H}_{\mathrm{D}}(\Gamma)}^{2}+\imath \alpha \llbracket \mathfrak{v}, \overline{\mathfrak{v}} \rrbracket \quad \text { for } \alpha= \pm \omega .
\end{aligned}
$$


We shall assume that the scalar coefficient $\omega>0$, usually referred to as impedance, is fixed until the end of this article. From the above identity we deduce an expression for the difference between ingoing and outgoing traces.

Corollary 6.1. We have $\left\|\mathfrak{v}_{\mathrm{N}}+\imath \omega \mathrm{T}\left(\mathfrak{v}_{\mathrm{D}}\right)\right\|_{\mathbb{H}_{N}(\Gamma)}^{2}-\left\|\mathfrak{v}_{\mathrm{N}}-\imath \omega \mathrm{T}\left(\mathfrak{v}_{\mathrm{D}}\right)\right\|_{\mathbb{H}_{\mathrm{N}}(\Gamma)}^{2}=2 \imath \omega \llbracket \mathfrak{v}, \overline{\mathfrak{v}} \rrbracket$ for all $\mathfrak{v}=\left(\mathfrak{v}_{\mathrm{D}}, \mathfrak{v}_{\mathrm{N}}\right) \in \mathbb{H}(\Gamma)$.

So-called ingoing/outgoing Robin trace operators also play an important role in scattering theory so, in the present paragraph, we study these trace operators in more detail. Define $\tau_{ \pm}^{j}: \mathrm{H}^{1}\left(\Delta, \bar{\Omega}_{j}\right) \rightarrow \mathrm{H}^{-1 / 2}\left(\partial \Omega_{j}\right)$ by

$$
\begin{aligned}
\tau_{ \pm}^{j}(\phi) & :=\mu_{j} \tau_{\mathrm{N}}^{j}(\phi) \pm \imath \omega \mathrm{T}_{j}\left(\tau_{\mathrm{D}}^{j}(\phi)\right) \quad \text { for } \phi \in \mathrm{H}^{1}\left(\Delta, \bar{\Omega}_{j}\right), \\
\tau_{ \pm} & :=\operatorname{diag}_{j=0 \ldots \mathrm{J}}\left(\tau_{ \pm}^{j}\right) .
\end{aligned}
$$

Remark 6.1. It is crucial for the subsequent analysis that the impedance operator involved in the definition of $\tau_{ \pm}$coincides with the DtN map T defined in Section 3.4 that induces scalar products on $\mathbb{H}_{\mathrm{D}}(\Gamma)$ and $\mathbb{H}_{\mathrm{N}}(\Gamma)$.

The Robin trace operators can be considered for prescribing boundary data for the solution of wave equations in each subdomain. Due to the positivity of the DtN maps $\mathrm{T}_{j}$, the associated boundary value problems are systematically well posed.

Lemma 6.2. For any $g \in \mathrm{L}^{2}\left(\Omega_{j}\right)$ with bounded support, and any $h \in \mathrm{H}^{-1 / 2}\left(\partial \Omega_{j}\right)$, there exists a unique $\phi \in \mathrm{H}_{\mathrm{loc}}^{1}\left(\bar{\Omega}_{j}\right)$ such that $\operatorname{div}(\mu \nabla \phi)+\kappa^{2} \phi=g$ in $\Omega_{j}$, and $\tau_{-}^{j}(\phi)=h$ on $\partial \Omega_{j}$ (and $\phi$ is $\kappa_{0}$-outgoing if $j=0$ ).

The proof of the previous lemma is a basic exercise on variational formulations, so it is left to the reader. We need to introduce resolvent operators that solve Helmholtz equation in each subdomain with a prescribed outgoing Robin boundary trace, the operator $\mathrm{S}^{j}: \mathrm{H}^{-1 / 2}\left(\partial \Omega_{j}\right) \rightarrow \mathrm{H}^{-1 / 2}\left(\partial \Omega_{j}\right)$ defined by

$$
\begin{aligned}
\mathrm{S}^{j}\left(\tau_{-}^{j}(\phi)\right)=\tau_{+}^{j}(\phi) \quad & \text { for all } \phi \in \mathrm{H}_{\mathrm{loc}}^{1}\left(\bar{\Omega}_{j}\right) \text { satisfying } \\
& \operatorname{div}(\mu \nabla \phi)+\kappa^{2} \phi=0 \text { in } \Omega_{j}, \\
& \phi \kappa_{0}-\text { outgoing radiating for } j=0 .
\end{aligned}
$$

The following result, very similar to Lemma 4.1 of [28], was established in Lemma 3, Chapter 2 of [41]. We provide the proof for the sake of completeness.

Proposition 6.2. The operator $\mathrm{S}=\operatorname{diag}_{j=0 \ldots \mathrm{J}}\left(\mathrm{S}^{j}\right)$ continuously maps $\mathbb{H}_{\mathrm{N}}(\Gamma)$ into $\mathbb{H}_{\mathrm{N}}(\Gamma)$ and is non-expansive: for all $\mathfrak{p} \in \mathbb{H}_{\mathrm{N}}(\Gamma)$ we have

$$
\|\mathrm{S}(\mathfrak{p})\|_{\mathbb{H}_{\mathbb{N}}(\Gamma)} \leq\|\mathfrak{p}\|_{\mathbb{H}_{N}(\Gamma)} .
$$

Proof. Pick an arbitrary $\mathfrak{p}=\left(p^{j}\right)_{j=0}^{\mathrm{J}} \in \mathbb{H}_{\mathrm{N}}(\Gamma)$. Applying Lemma 6.2, there exist functions $\phi_{j} \in \mathrm{H}_{\text {loc }}^{1}\left(\bar{\Omega}_{j}\right)$ such that $\operatorname{div}\left(\mu \nabla \phi_{j}\right)+\kappa^{2} \phi_{j}=0$ in $\Omega_{j}$, and $\tau_{-}^{j}\left(\phi_{j}\right)=p^{j}$ on $\partial \Omega_{j}$ (and $\phi_{j}$ is $\kappa_{0}$-outgoing if $j=0$ ). Set $\mathfrak{v}=\left(\mathfrak{v}_{\mathrm{D}}, \mathfrak{v}_{\mathrm{N}}\right):=\left(\tau_{\mathrm{D}}^{j}\left(\phi_{j}\right), \mu_{j} \tau_{\mathrm{N}}^{j}\left(\phi_{j}\right)\right)_{j=0, \ldots, \mathrm{J}}$, we have $\mathfrak{v}_{\mathrm{N}}-\imath \omega \mathrm{T}\left(\mathfrak{v}_{\mathrm{D}}\right)=\mathfrak{p}$ and $\mathfrak{v}_{\mathrm{N}}+\imath \omega \mathrm{T}\left(\mathfrak{v}_{\mathrm{D}}\right)=\mathrm{S}(\mathfrak{p})$. Since $\mathfrak{v} \in \mathscr{C}^{+}(\Gamma)$ by construction, combining Corollary 6.1 and Lemma 6.1 concludes the proof.

The previous result shows that the scattering operator $\mathrm{S}$ is a contraction but it is not a priori an isometry. In the context of problem (2.2), this is due to energy loss through radiation of waves toward infinity and absorption properties of the propagation medium (positive imaginary part of $\kappa^{2}$ ).

\section{ReFormulation of THE SCATTERING PROBLEM}

In the present section we describe a reformulation of the scattering problem (2.2) as an equivalently well posed problem. 


\subsection{Derivation of the formulation}

To take account of the right hand side $f \in \mathrm{L}^{2}\left(\mathbb{R}^{d}\right)$, we introduce the offset function $\phi_{f} \in \mathrm{L}_{\text {loc }}^{2}\left(\mathbb{R}^{d}\right)$ whose restriction to each subdomain $\left.\phi_{f}\right|_{\Omega_{j}}$ belongs to $\mathrm{H}_{\text {loc }}^{1}\left(\bar{\Omega}_{j}\right)$ and is the unique solution to

$$
\begin{aligned}
& \operatorname{div}\left(\mu \nabla \phi_{f}\right)+\kappa^{2} \phi_{f}=-f \quad \text { in } \Omega_{j}, \\
& \phi_{f} \text { is } \kappa_{0} \text {-outgoing, } \\
& \tau_{-}^{j}\left(\phi_{f}\right)=0 \quad \forall j=0 \ldots \mathrm{J} .
\end{aligned}
$$

Well posedness of the problem above is a straightforward consequence of Lemma 6.2. We have now all the ingredients required for deriving a reformulation of our scattering problem (2.2).

Lemma 7.1. Assume that $u \in \mathrm{H}_{\mathrm{loc}}^{1}\left(\mathbb{R}^{d}\right)$ is the unique solution to (2.2), and denote $\mathfrak{f}:=\Pi\left(\tau_{+}\left(\phi_{f}\right)\right)$ with $\phi_{f}$ defined by (7.1). Then the tuple of traces $\mathfrak{p}=\tau_{-}(u)$ satisfies

$$
\begin{aligned}
& \mathfrak{p} \in \mathbb{H}_{N}(\Gamma) \text { and } \\
& \mathfrak{p}-(\Pi \cdot S) \mathfrak{p}=\mathfrak{f} .
\end{aligned}
$$

Proof. If $u \in \mathrm{H}_{\mathrm{loc}}^{1}\left(\mathbb{R}^{d}\right)$ refers to the unique solution to $(2.2)$ then $\left(\tau_{\mathrm{D}}^{j}(u), \mu_{j} \tau_{\mathrm{N}}^{j}(u)\right)_{j=0 \ldots \mathrm{J}} \in \mathbb{X}(\Gamma)$ so, according to Proposition 5.4, we have $\tau_{-}(u)=\Pi\left(\tau_{+}(u)\right)$. In addition, the function $u-\phi_{f}$ solves an homogenous Helmholtz equation in each subdomain i.e. $\left(\operatorname{div}(\mu \nabla \cdot)+\kappa^{2}\right)\left(u-\phi_{f}\right)=0$ in $\Omega_{j}$ for each $j=0 \ldots \mathrm{J}$ and $u-\phi_{f}$ is $\kappa_{0}$-outgoing radiating, so $\left(\tau_{\mathrm{D}}^{j}\left(u-\phi_{f}\right), \mu_{j} \tau_{\mathrm{N}}^{j}\left(u-\phi_{f}\right)\right)_{j=0 \ldots \mathrm{J}} \in \mathscr{C}^{+}(\Gamma)$. As a consequence we have $\tau_{+}\left(u-\phi_{f}\right)=\mathrm{S} \cdot \tau_{-}\left(u-\phi_{f}\right)=$ $\mathrm{S} \cdot \tau_{-}(u)$. Thus we conclude that $\tau_{-}(u)=\Pi \mathrm{S}\left(\tau_{-}(u)\right)+\Pi \tau_{+}\left(\phi_{f}\right)$.

The structure of this new formulation is strikingly close to standard Optimised Schwarz Methods (OSM). This appears clearly when comparing (7.2) with Section 2 in [21], see in particular formula (45) and (51) of this reference.

Here also (7.2) appears adapted to domain decomposition. In the operator Id $-\Pi \cdot \mathrm{S}$, the factor $\mathrm{S}$ is blockdiagonal, each block being associated to a different subdomain, so that matrix-vector product is trivially parallelisable. Of course, each block of S involves a DtN operator. Once equation (7.2) has been solved numerically and that $\mathfrak{p}=\left(p_{j}\right)_{j=0 \ldots \mathrm{J}}$ has been computed, the solution to the boundary value problem (2.2) can be recovered in each $\Omega_{j}$ separately by solving the following local problems

$$
\begin{aligned}
& \operatorname{div}(\mu \nabla u)+\kappa^{2} u=-f \quad \text { in } \Omega_{j}, \\
& u \text { is } \kappa_{0} \text {-outgoing, } \\
& \tau_{-}^{j}(u)=p_{j} \quad \forall j=0 \ldots \mathrm{J} .
\end{aligned}
$$

The main new feature of the formulation we present here is the transmission operator $\Pi$. Contrary to the exchange operator traditionally used in OSM, see e.g. formula (42) in [21], our transmission operator $\Pi$ is not local anymore. But it only involves exponentially decaying kernels, with a damping factor $\gamma$ that can be tuned, so that $\Pi$ can nevertheless be considered quasi-local. In addition, various techniques (H-matrices [6, 8, 39], Fast Multipole Method $[25,38]$ ) can be used to sparsify this operator further.

\subsection{Well-posedness of the new formulation}

Let us examine the properties of the operator $\mathrm{Id}-\Pi \cdot \mathrm{S}$ in detail. First of all $\Pi \cdot \mathrm{S}$ continuously maps $\mathbb{H}_{\mathrm{N}}(\Gamma)$ into $\mathbb{H}_{\mathrm{N}}(\Gamma)$. In addition, combining Corollary 5.1 and Proposition 6.2, we obtain a contractivity result.

Lemma 7.2. We have $\|\Pi \cdot \mathrm{S}(\mathfrak{p})\|_{\mathbb{H}_{N}(\Gamma)} \leq\|\mathfrak{p}\|_{\mathbb{H}_{N}(\Gamma)}$ for all $\mathfrak{p} \in \mathbb{H}_{N}(\Gamma)$. 
Coming back to Remarks 5.1 and 6.1 , we stress that the estimate above is made possible by the fact the very same operator $\mathrm{T}$ comes into play in the definition of both $\Pi$ and $\mathrm{S}$ (through the impedance trace operators $\tau_{ \pm}$).

A direct consequence of this property is that the numerical range of the operator $\mathrm{Id}-\Pi \cdot \mathrm{S}$ is located in the complex right-half plane $\mathbb{C}_{+}:=\{z \in \mathbb{C}, \Re e\{z\} \geq 0\}$. This is definitely an interesting feature from the perspective of linear solvers. Next this operator is also one-to-one.

Proposition 7.1. $\operatorname{ker}(\mathrm{Id}-\Pi \cdot \mathrm{S})=\{0\}$.

Proof. Consider a $\mathfrak{p}=\left(p^{j}\right)_{j=0}^{J} \in \mathbb{H}_{N}(\Gamma)$ satisfying $\mathfrak{p}=\Pi \mathrm{S}(\mathfrak{p})$. Consider the function $v \in \mathrm{L}_{\text {loc }}^{2}\left(\mathbb{R}^{d}\right)$ such that, its restriction in each subdomain $\left.v\right|_{\Omega_{j}}$ belongs to $\mathrm{H}_{\mathrm{loc}}^{1}\left(\bar{\Omega}_{j}\right)$ and satisfies $\operatorname{div}(\mu \nabla v)+\kappa^{2} v=0$ in $\Omega_{j}, v$ is $\kappa_{0^{-}}$ outgoing and $\tau_{-}^{j}(v)=p^{j}$. By construction we have $\tau_{-}(v)=\mathfrak{p}$ and $\tau_{+}(v)=\mathrm{S}(\mathfrak{p})$. Setting $\mathfrak{v}=\left(\mathfrak{v}_{\mathrm{D}}, \mathfrak{v}_{\mathrm{N}}\right):=$ $\left(\tau_{\mathrm{D}}^{j}(v), \mu_{j} \tau_{\mathrm{N}}^{j}(v)\right)_{j=0 \ldots \mathrm{J}}$, we have $0=\mathfrak{p}-\Pi \mathrm{S}(\mathfrak{p})=\mathfrak{v}_{\mathrm{N}}-\imath \omega \mathrm{T}\left(\mathfrak{v}_{\mathrm{D}}\right)-\Pi\left(\mathfrak{v}_{\mathrm{N}}+\imath \omega \mathrm{T}\left(\mathfrak{v}_{\mathrm{D}}\right)\right)$. Hence, applying Proposition 5.4, we deduce that $\mathfrak{v} \in \mathbb{X}(\Gamma)$. Since, on the other hand, we have $\mathfrak{v} \in \mathscr{C}^{+}(\Gamma)$ by construction, we conclude that $\mathfrak{v} \in \mathscr{C}^{+}(\Gamma) \cap \mathbb{X}(\Gamma)=\{0\}$ according to Proposition 6.1. Hence $\mathfrak{p}=\mathfrak{v}_{\mathrm{N}}-\imath \omega \mathrm{T}\left(\mathfrak{v}_{\mathrm{D}}\right)=0$.

The next theorem is the main novelty of the present contribution. It shows that (7.2) offers a new strongly coercive formulation of the scattering problem (2.2). As discussed in [46], this directly contradicts the widespread belief that harmonic wave propagation systematically leads to sign indefinite formulations.

Theorem 7.1. There exists $\alpha>0$ such that $\Re e\left\{((\operatorname{Id}-\Pi \cdot \mathrm{S}) \mathfrak{p}, \mathfrak{p})_{\mathbb{H}_{N}(\Gamma)}\right\} \geq \alpha\|\mathfrak{p}\|_{\mathbb{H}_{N}(\Gamma)}^{2}$ for all $\mathfrak{p} \in \mathbb{H}_{N}(\Gamma)$.

Proof. We need first to introduce a few notations that we shall use only for this proof. According to Proposition 6.1, there exists a bounded projection operator Q : $\mathbb{H}(\Gamma) \rightarrow \mathbb{H}(\Gamma)$ with range $(\mathrm{Q})=\mathscr{C}^{+}(\Gamma)$ and $\operatorname{ker}(\mathrm{Q})=\mathbb{X}(\Gamma)$. For convenience, we set

$$
\begin{aligned}
& \|\mathrm{Q}\|_{\omega}:=\sup _{\mathfrak{v} \in \mathbb{H}(\Gamma) \backslash\{0\}}\|\mathrm{Q}(\mathfrak{v})\|_{\omega} /\|\mathfrak{v}\|_{\omega} \\
& \text { where }\|\mathfrak{v}\|_{\omega}^{2}:=\left\|\mathfrak{v}_{\mathrm{N}}\right\|_{\mathbb{H}_{N}(\Gamma)}^{2}+\omega^{2}\left\|\mathfrak{v}_{\mathrm{D}}\right\|_{\mathbb{H}_{\mathrm{D}}(\Gamma)}^{2}
\end{aligned}
$$

Because $\omega>0$ is a simple fixed positive constant, \|\|$_{\omega}$ and \|\|$_{\mathbb{H}(\Gamma)}$ are equivalent norms, and continuity of the projection $\mathrm{Q}$ is exactly equivalent to the boundedness of $\|\mathrm{Q}\|_{\omega}$. We shall also consider the bounded orthogonal projectors $\mathrm{P}_{ \pm}: \mathbb{H}_{\mathrm{N}}(\Gamma) \rightarrow \mathbb{H}_{\mathrm{N}}(\Gamma)$ defined by

$$
\mathrm{P}_{ \pm}=(\mathrm{Id} \pm \Pi) / 2
$$

Now pick an arbitrary $\mathfrak{p} \in \mathbb{H}_{\mathrm{N}}(\Gamma)$. Set $\mathfrak{f}:=(\mathrm{Id}-\Pi \mathrm{S}) \mathfrak{p}$, and define $\mathfrak{g}_{\mathrm{D}}:=\imath \omega^{-1} \mathrm{~T}^{-1}(\mathrm{Id}+\Pi) \mathfrak{f} / 4$ and $\mathfrak{g}_{\mathrm{N}}:=$ $(\operatorname{Id}-\Pi) \mathfrak{f} / 4$ and $\mathfrak{g}:=\left(\mathfrak{g}_{\mathrm{D}}, \mathfrak{g}_{\mathrm{N}}\right) \in \mathbb{H}(\Gamma)$. The tuple of traces $\mathfrak{u}=\mathrm{Q}(\mathfrak{g}) \in \mathscr{C}^{+}(\Gamma)$ satisfies $\mathfrak{g}-\mathfrak{u} \in \mathbb{X}(\Gamma)$ so, applying Proposition 5.4, we also have $\mathfrak{u}_{\mathrm{N}}-\mathfrak{g}_{\mathrm{N}}-\imath \omega \mathrm{T}\left(\mathfrak{u}_{\mathrm{D}}-\mathfrak{g}_{\mathrm{D}}\right)=\Pi\left(\mathfrak{u}_{\mathrm{N}}-\mathfrak{g}_{\mathrm{N}}+\imath \omega \mathrm{T}\left(\mathfrak{u}_{\mathrm{D}}-\mathfrak{g}_{\mathrm{D}}\right)\right)$ which rewrites

$$
\begin{aligned}
\mathfrak{u}_{\mathrm{N}}-\imath \omega \mathrm{T}\left(\mathfrak{u}_{\mathrm{D}}\right)-\Pi\left(\mathfrak{u}_{\mathrm{N}}+\imath \omega \mathrm{T}\left(\mathfrak{u}_{\mathrm{D}}\right)\right) \\
=(\mathrm{Id}-\Pi) \mathfrak{g}_{\mathrm{N}}-\imath \omega(\mathrm{Id}+\Pi) \mathrm{T}\left(\mathfrak{g}_{\mathrm{D}}\right) \\
=\mathrm{P}_{-}^{2} \mathfrak{f}+\mathrm{P}_{+}^{2} \mathfrak{f}=\left(\mathrm{P}_{-}+\mathrm{P}_{+}\right) \mathfrak{f}=\mathfrak{f} .
\end{aligned}
$$

Due to the continuity of $\mathrm{Q}$, we obviously have $\|\mathfrak{u}\|_{\omega} \leq\|\mathrm{Q}\|_{\omega} \cdot\|\mathfrak{g}\|_{\omega}$, where $\|\mathrm{Q}\|_{\omega}$ is defined with (7.4). On the other hand multiplying (7.6) on the left by $\mathrm{P}_{ \pm}$we obtain

$$
\begin{aligned}
& \mathrm{P}_{+} \mathrm{T}\left(\mathfrak{u}_{\mathrm{D}}\right)=\imath \omega^{-1} \mathrm{P}_{+}(\mathfrak{f}) / 2=\mathrm{T}\left(\mathfrak{g}_{\mathrm{D}}\right) \\
& \mathrm{P}_{-}\left(\mathfrak{u}_{\mathrm{N}}\right)=\mathrm{P}_{-}(\mathfrak{f}) / 2=\mathfrak{g}_{\mathrm{N}} \\
& \Rightarrow \quad\|\mathfrak{g}\|_{\omega}^{2}=\omega^{2}\left\|\mathrm{P}_{+} \mathrm{T}\left(\mathfrak{u}_{\mathrm{D}}\right)\right\|_{\mathbb{H}_{\mathrm{N}}(\Gamma)}^{2}+\left\|\mathrm{P}_{-}\left(\mathfrak{u}_{\mathrm{N}}\right)\right\|_{\mathbb{H}_{\mathrm{N}}(\Gamma)}^{2}
\end{aligned}
$$


which shows that $\|\mathfrak{u}\|_{\omega} \leq\|\mathrm{Q}\|_{\omega}\left(\omega^{2}\left\|\mathrm{P}_{+} \mathrm{T}\left(\mathfrak{u}_{\mathrm{D}}\right)\right\|_{\mathbb{H}_{N}(\Gamma)}^{2}+\left\|\mathrm{P}_{-}\left(\mathfrak{u}_{\mathrm{N}}\right)\right\|_{\mathbb{H}_{N}(\Gamma)}^{2}\right)$. Since $\mathfrak{u} \in \operatorname{range}(\mathrm{Q})=\mathscr{C}^{+}(\Gamma)$ we have $\mathfrak{u}_{\mathrm{N}}+\imath \omega \mathrm{T}\left(\mathfrak{u}_{\mathrm{D}}\right)=\mathrm{S}\left(\mathfrak{u}_{\mathrm{N}}-\imath \omega \mathrm{T}\left(\mathfrak{u}_{\mathrm{D}}\right)\right)$ and, as a consequence, (7.6) implies (Id $\left.-\Pi \mathrm{S}\right)\left(\mathfrak{u}_{\mathrm{N}}-\imath \omega \mathrm{T}\left(\mathfrak{u}_{\mathrm{D}}\right)\right)=\mathfrak{f}$ hence, according to Proposition $7.1, \mathfrak{p}=\mathfrak{u}_{\mathrm{N}}-\imath \omega \mathrm{T}\left(\mathfrak{u}_{\mathrm{D}}\right)$, which leads to the estimate

$$
\|\mathfrak{p}\|_{\mathbb{H}_{N}(\Gamma)}^{2} / 2 \leq\|\mathfrak{u}\|_{\omega}^{2} \leq\|\mathrm{Q}\|_{\omega}^{2}\|\mathfrak{g}\|_{\omega}^{2}
$$

Since the projectors $\mathrm{P}_{ \pm}$are orthogonal for the scalar product $(\cdot, \cdot)_{\mathbb{H}_{\mathrm{N}}(\Gamma)}$ we obtain

$$
\begin{aligned}
\frac{1}{2}(\mathfrak{p}- & \Pi S(\mathfrak{p}), \mathfrak{p})_{\mathbb{H}_{N}(\Gamma)} \\
= & \frac{1}{2}\left(\mathfrak{u}_{\mathrm{N}}-\imath \omega \mathrm{T}\left(\mathfrak{u}_{\mathrm{D}}\right)-\Pi\left(\mathfrak{u}_{\mathrm{N}}+\imath \omega \mathrm{T}\left(\mathfrak{u}_{\mathrm{D}}\right)\right), \mathfrak{u}_{\mathrm{N}}-\imath \omega \mathrm{T}\left(\mathfrak{u}_{\mathrm{D}}\right)\right)_{\mathbb{H}_{N}(\Gamma)} \\
= & \left(\mathrm{P}_{-}\left(\mathfrak{u}_{\mathrm{N}}\right)-\imath \omega \mathrm{P}_{+} \mathrm{T}\left(\mathfrak{u}_{\mathrm{D}}\right), \mathfrak{u}_{\mathrm{N}}-\imath \omega \mathrm{T}\left(\mathfrak{u}_{\mathrm{D}}\right)\right)_{\mathbb{H}_{\mathrm{N}}(\Gamma)} \\
= & \left\|\mathrm{P}_{-}\left(\mathfrak{u}_{\mathrm{N}}\right)\right\|_{\mathbb{H}_{N}(\Gamma)}^{2}+\omega^{2}\left\|\mathrm{P}_{+} \mathrm{T}\left(\mathfrak{u}_{\mathrm{D}}\right)\right\|_{\mathbb{H}_{N}(\Gamma)}^{2} \\
& -\imath \omega\left(\mathrm{P}_{+} \mathrm{T}\left(\mathfrak{u}_{\mathrm{D}}\right), \mathfrak{u}_{\mathrm{N}}\right) \mathbb{H}_{\mathbb{N}}(\Gamma)+\imath \omega\left(\mathrm{P}_{-}\left(\mathfrak{u}_{\mathrm{N}}\right), \mathrm{T}\left(\mathfrak{u}_{\mathrm{D}}\right)\right)_{\mathbb{H}_{\mathrm{N}}(\Gamma)} .
\end{aligned}
$$

Using the identity obtained in (7.7) to replace $\left\|\mathrm{P}_{-}\left(\mathfrak{u}_{\mathrm{N}}\right)\right\|_{\mathbb{H}_{N}(\Gamma)}^{2}+\omega^{2}\left\|\mathrm{P}_{+} \mathrm{T}\left(\mathfrak{u}_{\mathrm{D}}\right)\right\|_{\mathbb{H}_{N}(\Gamma)}^{2}$ in the identity above, using that $\mathrm{P}_{ \pm}=(\operatorname{Id} \pm \Pi) / 2$, and observing that $(\mathrm{T}(\mathfrak{v}), \mathfrak{p})_{\mathbb{H}_{\mathbb{N}}(\Gamma)}=\langle\langle\mathfrak{v}, \overline{\mathfrak{p}}\rangle\rangle$, we obtain

$$
\begin{aligned}
& \frac{1}{2}(\mathfrak{p}-\Pi S(\mathfrak{p}), \mathfrak{p})_{\mathbb{H}_{N}(\Gamma)} \\
& =\|\mathfrak{g}\|_{\omega}^{2}-(\imath \omega / 2)\left\langle\left\langle\mathfrak{u}_{\mathrm{D}}, \overline{\mathfrak{u}}_{\mathrm{N}}\right\rangle\right\rangle+(\imath \omega / 2)\left\langle\left\langle\mathfrak{u}_{\mathrm{N}}, \overline{\mathfrak{u}}_{\mathrm{D}}\right\rangle\right\rangle \\
& -(\imath \omega / 2)\left(\Pi T\left(\mathfrak{u}_{\mathrm{D}}\right), \mathfrak{u}_{\mathrm{N}}\right)_{\mathbb{H}_{\mathrm{N}}(\Gamma)}-(\imath \omega / 2)\left(\Pi\left(\mathfrak{u}_{\mathrm{N}}\right), \mathrm{T}\left(\mathfrak{u}_{\mathrm{D}}\right)\right)_{\mathbb{H}_{\mathrm{N}}(\Gamma)} \\
& =\|\mathfrak{g}\|_{\omega}^{2}-(\imath \omega / 2) \llbracket \mathfrak{u}, \overline{\mathfrak{u}} \rrbracket-\imath \omega \Re e\left\{\left(\Pi T\left(\mathfrak{u}_{\mathrm{D}}\right), \mathfrak{u}_{\mathrm{N}}\right)_{\mathbb{H}_{N}(\Gamma)}\right\} .
\end{aligned}
$$

Using Lemma 6.1, the real part of the previous identity is bounded from below by $\Re e\left\{(\mathfrak{p}-\Pi S(\mathfrak{p}), \mathfrak{p})_{\mathbb{H}_{N}}(\Gamma)\right\} \geq$ $2\|\mathfrak{g}\|_{\omega}^{2}$. We conclude by using (7.8).

Lax-Milgram lemma combined with the previous theorem yields bijectivity of Id - ПS as an obvious outcome.

Corollary 7.1. The operator $\mathrm{Id}-\Pi \mathrm{S}: \mathbb{H}_{\mathrm{N}}(\Gamma) \rightarrow \mathbb{H}_{\mathrm{N}}(\Gamma)$ is an isomorphism.

\subsection{Solution strategy}

Let us briefly discuss how, in practice, to solve (7.2) i.e. an equation of the form $\mathfrak{p}-\Pi \cdot S(\mathfrak{p})=\mathfrak{f}$. First of all, since $\Pi^{2}=\mathrm{Id}$, this equation can be transformed into $(\Pi-S) \mathfrak{p}=\Pi(\mathfrak{f})=\tau_{+}\left(\phi_{f}\right)$ which is practically more convenient as it avoids handling a product of operators. A general Krylov solver such as GMRes could be considered for solving this equation. We refer the reader to Chapter 6 of [51] for more details on this solver.

Convergence of Richardson's linear solver. An alternative more straightforward strategy relies on Richardson's iterative method ([51], Chap. 6, [1], Sect. 9.1) that writes

$$
\mathfrak{p}^{n+1}=(1-\beta) \mathfrak{p}^{(n)}+\beta \Pi S \cdot \mathfrak{p}^{(n)}+\beta \mathfrak{f}
$$

where $\beta \in(0,1)$ is a relaxation parameter. Following Theorem 7 and Remark 9 in [21], a rough estimate can be derived for the convergence of Richardson's linear solver in this case. Let $\mathfrak{p}^{\infty}$ refer to the unique solution to $(7.2)$ and set $\mathfrak{e}^{(n)}:=\mathfrak{p}^{\infty}-\mathfrak{p}^{(n)}$ so that $\mathfrak{e}^{(n+1)}=((1-\beta) \operatorname{Id}+\beta \Pi S) \mathfrak{e}^{(n)}$. Recall the convexity identity

$$
\begin{aligned}
\|(1-\beta) \mathbf{x}+\beta \mathbf{y}\|_{\mathbb{H}_{N}(\Gamma)}^{2}= & (1-\beta)\|\mathbf{x}\|_{\mathbb{H}_{N}(\Gamma)}^{2}+\beta\|\mathbf{y}\|_{\mathbb{H}_{N}(\Gamma)}^{2} \\
& -\beta(1-\beta)\|\mathbf{x}-\mathbf{y}\|_{\mathbb{H}_{N}(\Gamma)}^{2}
\end{aligned}
$$


which holds for any $\mathbf{x}, \mathbf{y} \in \mathbb{H}_{\mathrm{N}}(\Gamma)$ and any $\beta \in(0,1)$. In addition the coercivity estimate of Theorem 7.1 yields the lower bound $\|(\operatorname{Id}-\Pi S) \mathfrak{p}\|_{\mathbb{H}_{N}(\Gamma)} \geq \alpha\|\mathfrak{p}\|_{\mathbb{H}_{N}(\Gamma)} \forall \mathfrak{p} \in \mathbb{H}_{N}(\Gamma)$. Combining this lower bound with Lemma 7.2 and (7.12) thus yields

$$
\begin{aligned}
\left\|\mathfrak{e}^{(n+1)}\right\|_{\mathbb{H}_{\mathbb{N}}(\Gamma)=}^{2} & \left\|(1-\beta) \mathfrak{e}^{(n)}+\beta \Pi S \cdot \mathfrak{e}^{(n)}\right\|_{\mathbb{H}_{\mathbb{N}}(\Gamma)}^{2} \\
= & (1-\beta)\left\|\mathfrak{e}^{(n)}\right\|_{\mathbb{H}_{\mathbb{N}}(\Gamma)}^{2}+\beta\left\|\Pi \mathrm{S} \cdot \mathfrak{e}^{(n)}\right\|_{\mathbb{H}_{\mathbb{N}}(\Gamma)}^{2} \\
& -\beta(1-\beta)\left\|(\mathrm{Id}-\Pi S) \mathfrak{e}^{(n)}\right\|_{\mathbb{H}_{\mathbb{N}}(\Gamma)}^{2} \\
\leq & \left(1-\alpha^{2} \beta(1-\beta)\right)\left\|\mathfrak{e}^{(n)}\right\|_{\mathbb{H}_{N}(\Gamma)}^{2} .
\end{aligned}
$$

In this estimate, the convergence factor $\left(1-\alpha^{2} \beta(1-\beta)\right)^{1 / 2}<1$ is thus minimized for $\beta=1 / 2$ and takes the value $\left(1-(\alpha / 2)^{2}\right)^{1 / 2}$ in this case.

Acknowledgements. This work received support from the French National Research Agency (ANR) through the NonlocalDD project, grant ref. ANR-15-CE23-0017-01.

\section{REFERENCES}

[1] G. Allaire and S.M. Kaber, Numerical linear algebra. In: Vol. 55 of Texts in Applied Mathematics. New York, Springer (2008).

[2] X. Antoine and C. Geuzaine, Optimized Schwarz domain decomposition methods for scalar and vector Helmholtz equations. In: Modern Solvers for Helmholtz Problems. Birkhäuser/Springer, Basel (2017) 189-213.

[3] X. Antoine, Y. Boubendir and C. Geuzaine, A quasi-optimal non-overlapping domain decomposition algorithm for the Helmholtz equation. J. Comput. Phys. 231 (2012) 262-280.

[4] X. Antoine, C. Geuzaine and A. Modave, Corner treatment for high-order local absorbing boundary conditions in high-frequency acoustic scattering. J. Comput. Phys. 401 (2020) 109029.

[5] X. Antoine, C. Geuzaine, A. Modave and A. Royer, A non-overlapping domain decomposition method with high-order transmission conditions and cross-point treatment for Helmholtz problems. Comput. Methods Appl. Mech. Eng. 368 (2020) 113162 .

[6] M. Bebendorf, A means to efficiently solve elliptic boundary value problems. In: Vol. 63 of Hierarchical Matrices. Lecture Notes in Computational Science and Engineering. Springer-Verlag, Berlin (2008).

[7] A. Bendali and Y. Boubendir, Non-overlapping domain decomposition method for a nodal finite element method. Numer. Math. 103 (2006) 515-537.

[8] S. Börm, Efficient numerical methods for non-local operators: $\mathscr{H}^{2}$-matrix compression, algorithms and analysis. In: Vol. 14 of EMS Tracts in Mathematics. European Mathematical Society (EMS), Zürich (2010).

[9] Y. Boubendir, Techniques de Décomposition de Domaine et Méthodes d'Equations Intégrales. Ph.D. thesis, INSA of Toulouse (2002).

[10] X. Claeys, A single trace integral formulation of the second kind for acoustic scattering. Technical Report 2011-14, Seminar for Applied Mathematics, ETH Zürich, Switzerland (2011).

[11] X. Claeys, Quasi-local multitrace boundary integral formulations. Numer. Methods Part. Differ. Equ. 31 (2015) $2043-2062$.

[12] X. Claeys and R. Hiptmair, Electromagnetic scattering at composite objects: a novel multi-trace boundary integral formulation. ESAIM:M2AN 46 (2012) 1421-1445.

[13] X. Claeys and R. Hiptmair, Integral equations on multi-screens. Integral Equ. Oper. Theory 77 (2013) $167-197$.

[14] X. Claeys and R. Hiptmair, Multi-trace boundary integral formulation for acoustic scattering by composite structures. Comm. Pure Appl. Math. 66 (2013) 1163-1201.

[15] X. Claeys and E. Parolin, Robust treatment of cross points in Optimized Schwarz Methods. Preprint arXiv: 2003.06657 (2020).

[16] X. Claeys, R. Hiptmair and C. Jerez-Hanckes, Multitrace boundary integral equations. In: Direct and Inverse Problems in Wave Propagation and Applications. Selected Papers of the Workshop on Wave Propagation and Scattering, Inverse Problems and Applications in Energy and the Environment, Linz, Austria, November 21-25, 2011. de Gruyter, Berlin (2013) 51-100.

[17] X. Claeys, R. Hiptmair and E. Spindler, A second-kind Galerkin boundary element method for scattering at composite objects. BIT 55 (2015) 33-57.

[18] X. Claeys, R. Hiptmair and E. Spindler, Second kind boundary integral equation for multi-subdomain diffusion problems. Adv. Comput. Math. 43 (2017) 1075-1101.

[19] X. Claeys, R. Hiptmair and E. Spindler, Second-kind boundary integral equations for electromagnetic scattering at composite objects. Comput. Math. Appl. $\mathbf{7 4}$ (2017) 2650-2670.

[20] X. Claeys, B. Thierry and F. Collino, Integral equation based optimized schwarz method for electromagnetics. In: Domain Decomposition Methods in Science and Engineering XXIV, edited by P.E. Bjørstad, S.C. Brenner, L. Halpern, H.H. Kim, R. Kornhuber, T. Rahman and O.B. Widlund. Springer International Publishing, Cham (2018) 187-194.

[21] F. Collino, S. Ghanemi and P. Joly, Domain decomposition method for harmonic wave propagation: a general presentation. Comput. Methods Appl. Mech. Engrg. 184 (2000) 171-211. 
[22] D. Colton and R. Kress, Inverse acoustic and electromagnetic scattering theory, 2nd edition. In: Vol. 93 of Applied Mathematical Sciences. Springer, New York (2013).

[23] D. Colton and R. Kress, Integral equation methods in scattering theory. In: Vol. 72 of Classics in Applied Mathematics. Society for Industrial and Applied Mathematics (SIAM), Philadelphia, PA (2013).

[24] M. Costabel, Boundary integral operators on Lipschitz domains: elementary results. SIAM J. Math. Anal. 19 (1988) $613-626$.

[25] E. Darve, The fast multipole method: numerical implementation. J. Comput. Phys. 160 (2000) 195-240.

[26] B. Després, Décomposition de domaine et problème de Helmholtz. C. R. Acad. Sci. Paris Sér. I Math. 311 (1990) 313-316.

[27] B. Després, Domain decomposition method and the Helmholtz problem. In: Mathematical and Numerical Aspects of Wave Propagation Phenomena (Strasbourg, 1991). SIAM, Philadelphia, PA (1991) 44-52.

[28] B. Després, Méthodes de décomposition de domaine pour les problèmes de propagation d'ondes en régime harmonique. Le théorème de Borg pour l'équation de Hill vectorielle. Thèse, Institut National de Recherche en Informatique et en Automatique (INRIA), Rocquencourt, 1991, Université de Paris IX (Dauphine), Paris (1991).

[29] B. Després, Domain decomposition method and the Helmholtz problem. II. In: Second International Conference on Mathematical and Numerical Aspects of Wave Propagation (Newark, DE, 1993). SIAM, Philadelphia, PA (1993) $197-206$.

[30] B. Després, A. Nicolopoulos and B. Thierry, Corners and stable optimized domain decomposition methods for the Helmholtz problem. Preprint https://hal.archives-ouvertes.fr/hal-02612368 (2020).

[31] V. Dolean, P. Jolivet and F. Nataf, An Introduction to Domain Decomposition Methods. Algorithms, Theory, and Parallel Implementation. Society for Industrial and Applied Mathematics (SIAM), Philadelphia, PA (2015).

[32] M. El Bouajaji, X. Antoine and C. Geuzaine, Approximate local magnetic-to-electric surface operators for time-harmonic Maxwell's equations. J. Comput. Phys. 279 (2014) 241-260.

[33] M. El Bouajaji, B. Thierry, X. Antoine and C. Geuzaine, A quasi-optimal domain decomposition algorithm for the timeharmonic Maxwell's equations. J. Comput. Phys. 294 (2015) 38-57.

[34] M. Gander and F. Kwok, On the applicability of Lions' energy estimates in the analysis of discrete optimized Schwarz methods with cross points. In: Vol. 91 of Domain Decomposition Methods in Science and Engineering XX. Lect. Notes Comput. Sci. Eng. Springer, Heidelberg (2013) 475-483.

[35] M. Gander and K. Santugini, Cross-points in domain decomposition methods with a finite element discretization. Electron. Trans. Numer. Anal. 45 (2016) 219-240.

[36] M.J. Gander and H. Zhang, A class of iterative solvers for the Helmholtz equation: factorizations, sweeping preconditioners, source transfer, single layer potentials, polarized traces, and optimized Schwarz methods. SIAM Rev. 61 (2019) 3-76.

[37] L. Greengard and J.-Y. Lee, Stable and accurate integral equation methods for scattering problems with multiple material interfaces in two dimensions. J. Comput. Phys. 231 (2012) 2389-2395.

[38] L. Greengard and V. Rokhlin, A new version of the fast multipole method for the Laplace equation in three dimensions. In: Vol. 6 of Acta Numer. Cambridge Univ. Press, Cambridge (1997) 229-269.

[39] W. Hackbusch, Hierarchical matrices: algorithms and analysis. In: Vol. 49 of Springer Series in Computational Mathematics. Springer, Heidelberg (2015).

[40] U. Langer and O. Steinbach, Boundary element tearing and interconnecting methods. Computing 71 (2003) 205-228.

[41] M. Lecouvez, Iterative methods for domain decomposition without overlap with exponential convergence for the Helmholtz equation. Ph.D thesis, Ecole Polytechnique (2015).

[42] M. Lecouvez, B. Stupfel, P. Joly and F. Collino, Quasi-local transmission conditions for non-overlapping domain decomposition methods for the Helmholtz equation. C. R. Phys. 15 (2014) 403-414.

[43] R. Leis, Initial-Boundary Value Problems in Mathematical Physics, edited by B.G. Teubner, Stuttgart. John Wiley \& Sons, Ltd., Chichester (1986).

[44] P.-L. Lions, On the Schwarz alternating method III. A variant for nonoverlapping subdomains. In: Vol. 22 of Third International Symposium on Domain Decomposition Methods for Partial Differential Equations. SIAM (1989).

[45] W. McLean, Strongly Elliptic Systems and Boundary Integral Equations. Cambridge University Press, Cambridge (2000).

[46] A. Moiola and E.A. Spence, Is the Helmholtz equation really sign-indefinite? SIAM Rev. 56 (2014) 274-312.

[47] F.W.J. Olver, D.W. Lozier, R.F. Boisvert and C.W. Clark, NIST handbook of mathematical functions. In: U.S. Department of Commerce, National Institute of Standards and Technology. Cambridge University Press, Cambridge, Washington, DC (2010).

[48] C. Pechstein, Finite and boundary element tearing and interconnecting solvers for multiscale problems. In: Vol. 90 of Lecture Notes in Computational Science and Engineering. Springer, Heidelberg (2013).

[49] Z. Peng, K.-H. Lim and J.-F. Lee, Computations of electromagnetic wave scattering from penetrable composite targets using a surface integral equation method with multiple traces. IEEE Trans. Antennas Propag. 61 (2013) 256-270.

[50] Z. Peng, K.-H. Lim and J.-F. Lee, A boundary integral equation domain decomposition method for electromagnetic scattering from large and deep cavities. (English summary) J. Comput. Phys. 280 (2015) 626-642.

[51] Y. Saad, Iterative Methods for Sparse Linear Systems, 2nd edition. SIAM Society for Industrial and Applied Mathematics, Philadelphia, PA (2003).

[52] S. Sauter and C. Schwab, Boundary element methods. In: Vol. 39 of Springer Series in Computational Mathematics. SpringerVerlag, Berlin (2011).

[53] O. Steinbach, Numerical Approximation Methods for Elliptic Boundary Value Problems. Finite and Boundary Elements. Springer, New York (2008).

[54] A. Toselli and O. Widlund, Domain decomposition methods - algorithms and theory. In: Vol. 34 of Springer Series in Computational Mathematics. Springer-Verlag, Berlin (2005). 
[55] A. Vion and C. Geuzaine, Improved sweeping preconditioners for domain decomposition algorithms applied to time-harmonic Helmholtz and Maxwell problems. ESAIM: Proc. Surv. 61 (2018) 93-111.

[56] T. von Petersdorff, Boundary integral equations for mixed Dirichlet, Neumann and transmission problems. Math. Methods Appl. Sci. 11 (1989) 185-213. 